\title{
Cambios recientes en la sistemática y taxonomía de murciélagos Neotropicales (Mammalia: Chiroptera)
}

Sergio Solari ${ }^{1,2,3 *}$, y Víctor Martínez-Arias ${ }^{2}$

Introduction: Conservation of biodiversity requires an accurate accounting of the entities (taxa) comprising that diversity, the species. In an ideal system, each entity would have a unique and unequivocal name that would integrate information from other disciplines within a universal concept to ease the communication. In spite of being a comparatively well-known group, the number of recognized species of Neotropical bats continues to increase each year, making the organization and management of this knowledge difficult for the non-taxonomist. Here, we review and detail the most recent proposals in regard to the taxonomy of Neotropical bats to make this information more easily accessible to the non-taxonomist.

Methods: We used the list of species (and subspecies) recognized in the last edition of "Mammal Species of the World" (2005) as a starting point. From there, we gathered all relevant papers dealing with taxonomy of Neotropical bat species. We delimited the Neotropical region from the lowlands of tropical Mexico to Tierra del Fuego, including the Caribbean. We did not include fossil, extinct or unnamed species.

Results: Since 2005, the number of taxonomic changes (Table 1) has resulted in a net increase of 75 species and eigth genera, with a regional diversity of 380 species in 98 genera (Table 2). This richness increase includes newly recognized taxa (split from previously valid genera or species, often listed as junior synonyms) as well as previously unknown species, discovered in the field or at museum collections (Figure 2). Whereas some genera have increased their species richness by almost $100 \%$, the average increase is around two species per genus.

Discussion: The use of molecular genetics in systematics research, with an emphasis on recognition of reciprocally monophyletic groups, has contributed the most to the increase in new names now recognized. Although these revisions may appear to the non-taxonomist as a sign of taxonomic instability, we emphasize that the goal of these studies is to facilitate the integration of genetic, morphological, and biogeographic data to improve our knowledge of these species. We anticipate that further advances will continue as we increase exploration and research, together with the use of multiple sources of taxonomic information. All these data should enhance confidence and support for these results, and hopefully a broader agreement among Neotropical bat researchers.

Key words: taxonomic update, biodiversity, species, genera, nomenclature.

\section{Resumen}

La conservación de la biodiversidad requiere un conocimiento preciso y actualizado de los entes más fundamentales que la constituyen, las especies. En una taxonomía perfecta, cada entidad tendría un nombre único e inequívoco que integrando información de

\footnotetext{
'Instituto de Biología, Universidad de Antioquia, Medellín, Colombia. Calle 70 No. 52-21, Medellín AA 1226 Antioquia, Colombia. Email: solari.udea@gmail.com (SS).

${ }^{2}$ Grupo Mastozoología y Colección Teriológica, Universidad de Antioquia; Medellín, Colombia. Calle 70 No. 52-21, Medellín AA 1226. Antioquia, Colombia. Email: vmartinezarias@gmail.com

${ }^{3}$ Chiroptera Specialist Group, Species Survival Commission, IUCN.

${ }^{*}$ Corresponding author
} 
otras disciplinas biológicas, facilitaría una efectiva comunicación. A pesar de ser un grupo relativamente bien estudiado, el número de especies reconocidas de murciélagos (Chiroptera) Neotropicales muestra continuos incrementos que dificultan organizar y manejar este conocimiento. En este trabajo, intentamos consolidar y mostrar las propuestas más recientes en la taxonomía de murciélagos Neotropicales para que esta información sea más accesible a los investigadores que no son taxónomos al momento de plantear nuevos estudios o discutir sus resultados. Nuestra lista actualizada incluye 380 especies en 98 géneros. Tomando como punto de partida las especies del listado del 2005, el número de cambios en estatus taxonómico resulta en un incremento neto de ocho géneros y 75 especies. El método más empleado para reconocer estos géneros y especies adicionales ha sido el criterio de monofilia, el cual supone que cada nombre específico o genérico incluye sólo descendientes de un ancestro único. Aunque estas revisiones pueden interpretarse como ejemplos de la falta de consenso sistemático y la consecuente inestabilidad taxonómica, debemos enfatizar que el objetivo final de estos estudios es facilitar la integración de datos genéticos, morfológicos, y biogeográficos para mejorar el conocimiento global en estas especies.

Palabras clave: actualización taxonómica, biodiversidad, especies, géneros, nomenclatura.

La conservación de la biodiversidad requiere un conocimiento preciso y actualizado de los entes más fundamentales que la constituyen, las especies. Ello incluye tomar en cuenta aspectos como la ecología, distribución, y abundancia (o rareza). Sin embargo, en muy pocos casos esta información está disponible, actualizada y precisamente asociada a cada especie. La definición de especies, sus límites y sus relaciones respecto a otras especies es el sujeto de estudio de la disciplina que conocemos como sistemática (Wiley y Lieberman 2011). Bajo dicho sistema, aún imperfecto, pretendemos una taxonomía que refleje el mundo natural tal como lo percibimos, en la que cada entidad tenga un nombre único e inequívoco que integrando información de otras disciplinas biológicas, facilite una comunicación efectiva.

Para el Neotrópico, el número de especies reconocidas de mamíferos, uno de los grupos animales mejor estudiados, continua mostrando incrementos que dificultan organizar este conocimiento (Patterson 2000; Reeder et al. 2007; Ceballos y Ehrlich 2009). Nuevas especies son reconocidas como resultado de exploraciones de territorios poco estudiados (Reeder et al. 2007) y la aplicación de nuevos paradigmas para delimitar especies (de Queiroz 2007). Entre estos últimos, destaca el concepto filogenético de especie (Cracraft 1983; de Queiroz 1998; Velasco 2009), que ha sido usado especialmente en primates (Groves 2001) y de manera reciente en ungulados (Groves y Grubb 2011).

Si bien algunos autores (Zachos et al. 2013) critican el uso y supuesto abuso de este criterio, otros (Baker y Bradley 2006) afirman que esta aproximación permite reconocer filogrupos geográficos que en muchos casos se consideraban taxa distintos desde su descripción original, pero que se han usado como sinónimos menores o subespecies (ver Patterson 2000). 
Las principales observaciones hacia este uso del concepto filogenético de especie (Zachos et al. 2013) son que la evidencia para distinguir especies utiliza análisis simplistas de la extensión geográfica de variaciones morfológicas, y que esta extensión geográfica es solo cubierta en parte. Igualmente, su aplicación en la delimitación de especies no es estándar entre estudios, y en muchos casos parece arbitraria. Sin embargo, existen casos en los que esta evidencia incluye análisis morfológicos, morfométricos, cariotípicos, y genéticos, que permiten concluir que la diversidad de especies estaba realmente subestimada (Dayrat 2005; Padial et al. 2010). En este sentido, resulta claro que estudios robustos tienden a producir respuestas críticas y precisas para entender aspectos como evolución morfológica, reconstrucción biogeográfica, evaluaciones del estado de conservación, e incluso patrones espacio-temporales de diversificación (Barraclough y Nee 2001).

Sin embargo, más allá del respaldo de los datos y sus análisis, estos cambios taxonómicos resultan de difícil aceptación y aplicación para la totalidad de los investigadores, especialmente en el caso de aquéllos que trabajan en el área de la ecología y conservación (Agapow et al. 2004). A pesar de la importancia de los murciélagos tanto desde el punto de vista ecológico (Jones et al. 2009; Kunz et al. 2011) como epidemiológico (Drexler et al. 2012; Tong et al. 2013), el conocimiento taxonómico de muchas especies, especialmente en el muy diverso Neotrópico, no se mantiene estable. Por ello, cuando tratamos de integrar esta información biológica con los nombres en uso (nomenclatura) para facilitar la comunicación, el problema se magnifica.

Nuestra principal intención en esta nota es consolidar y mostrar las propuestas más recientes en la taxonomía de murciélagos Neotropicales para que los investigadores tengan esta información a mano al momento de plantear nuevos estudios, o discutir sus resultados. Usando ejemplos de estudios recientes (posteriores a 2004, fecha de cierre de la tercera edición de Mammal Species of the World; Wilson y Reeder 2005), mostramos que el respaldo de muestreos exhaustivos de especímenes y análisis detallados de su morfología continúan siendo importantes para estudios filogenéticos, evaluaciones

\section{Material} taxonómicas y actualización nomenclatural.

\section{y Métodos}

Como punto de partida usamos la lista de especies (y subespecies) reconocidas por Simmons (2005). Es importante notar que dicha lista solo incluyó actualizaciones hasta junio 2004. A partir de dicha lista, se hizo una recopilación de literatura relevante en términos de revisiones taxonómicas, descripciones o validaciones de especies, y en menor medida, listas regionales de especies. Nuestra lista de especies excluye especies extintas o fósiles, tanto en el conteo inicial (Simmons 2005) como en el final. El aspecto geográfico solo se consideró para confirmar la presencia de algunos taxa dentro de la región Neotropical (Ortega y Arita 1998).

Nuestra definición de la Región Neotropical es similar a la usada en estudios previos (Ortega y Arita 1998; Patterson 2000; Solari et al. 2012), abarcando el territorio desde las tierras bajas tropicales de México hasta Tierra del Fuego, e incluyendo el Caribe e islas asociadas. Debido a que los intervalos de las especies no coinciden exactamente con los límites entre regiones, algunas especies son arbitrariamente incluidas en la Región Neotropical a partir de estas distribuciones marginales. 
Cambios taxonómicos al nivel superior: subórdenes, infraórdenes y superfamilias. Simmons (2005) discutió pero no siguió la propuesta de Koopman $(1984,1994)$ que reconocía dos subórdenes, Megachiroptera (para los Pteropodidae) y Microchiroptera. La información disponible en ese momento sugería que las agrupaciones por encima del nivel de familia no eran soportadas en estudios moleculares. Dentro de los Microchiroptera, Koopman (1994) incluyó los infraórdenes Yinochiroptera (pre-maxilas móviles) y Yangochiroptera (pre-maxilas fijas). Para el Neotrópico, los Yinochiroptera incluyen a la superfamilia Emballonuroidea (Emballonuridae), mientras que los Yangochiroptera incluyen a los Noctilionoidea (Mormoopidae, Noctilionidae, Phyllostomidae) y Vespertilionoidea (Thyropteridae, Furipteridae, Natalidae, Molossidae, Vespertilionidae).

Al comprobarse la parafilia de Microchiroptera, Teeling et al. (2002) expandió la propuesta de Springer et al. (2001), con dos subórdenes: a) Yinpterochiroptera, que incluye a los Pteropodoidea y Rhinolophoidea; y b) Yangochiroptera, que incluye a las superfamilias Emballonuroidea, Noctilionoidea y Vespertilionoidea. Análisis subsecuentes (Teeling et al. 2003, 2005; Van Den Bussche y Hoofer 2004) afianzaron este ordenamiento, con los Noctilionoidea incluyendo a las familias Thyropteridae, Furipteridae, Noctilionidae, Mormoopidae y Phyllostomidae (además de Myzopodidae y Mystacinidae), y los Vespertilionoidea incluyendo a las familias Natalidae, Molossidae y Vespertilionidae.

Cambios taxonómicos a los niveles de familia y subfamilia. Simmons (2005) no consideró las conclusiones de revisiones taxonómicas anteriores para las familias Vespertilionidae (Hoofer y Van Den Bussche 2003) y Phyllostomidae (Baker et al. 2003). Posteriormente, fueron revisadas las familias Emballonuridae, con énfasis en la tribu Diclidurini (Lim et al. 2008b), y Molossidae, con énfasis en la subfamilia Molossinae (Ammerman et al. 2012).

Muchos grupos previamente reconocidos de Vespertilionidae fueron drásticamente cambiados por Hoofer y Van Den Bussche (2003), incluyendo el reconocimiento de Miniopteridae como una familia distinta, que incluye un único género (Miniopterus) cuya distribución se restringe al Viejo Mundo. Otro cambio relevante fue el reconocimiento de Myotis como único representante de su propia subfamilia (Myotinae), y separado de Vespertilioninae (sensu stricto). Esta última subfamilia incluye los restantes géneros del Neotrópico. Hoofer y Van Den Bussche (2003) también incluyeron otras conclusiones, cuyas implicaciones taxonómicas (algunas a nivel genérico, ver más abajo) no han sido completamente implementadas por autores subsecuentes.

La revisión de los Phyllostomidae (Baker et al. 2003) fue una nueva aproximación a la filogenia molecular presentada previamente (Baker et al. 2000), dadas las grandes diferencias con la revisión por Wetterer et al. (2000) usando solo morfología. El resultado más contrastante, y por tanto de difícil aceptación, fue la propuesta de reconocer 11 subfamilias donde sólo seis se reconocían tradicionalmente (Koopman 1994; Simmons 2005). Los cambios taxonómicos mayores propuestos por Baker et al. (2003) se deben a la polifilia de: a) los Phyllostominae, al incluir a Macrotinae, Micronycterinae, Lonchorhininae, "Glyphonycterinae" y Phyllostominae (sensu stricto); y b) los 
Carolliinae, dado que Carollia no es el grupo hermano de Rhinophylla (requiriéndose una nueva subfamilia para ésta). Para las dos nuevas subfamilias, Baker et al. (2003) propusieron nombres nuevos, pero estos no fueron descritos según los criterios del ICZN (1999) y por ello no están disponibles. Por otra parte, esta filogenia reforzó la distinción de Lonchophyllinae respecto a Glossophaginae, que incluye a los Brachyphyllinae y Phyllonycterinae de Simmons (2005), mientras que los Desmodontinae y Stenodermatinae no resultaron afectados en cuanto a géneros incluidos.

En el caso de los Emballonuridae, la revisión por Lim et al. (2008b) confirma que todos los géneros Neotropicales pertenecen a la tribu Diclidurini (dentro de Emballonurinae), tal como proponían Griffiths y Smith (1991), pero contrario al esquema de Jones y Hood (1993). En esta propuesta, se restringía Diclidurinae solo a Diclidurus y Cyttarops, mientras que los restantes géneros se consideraban parte de los Emballonurinae. De igual manera, la revisión de la subfamilia Molossinae por Ammerman et al. (2012) no cambió significativamente los grupos reconocidos en los Molossidae o la subfamilia, excepto por el soporte a la distinción de Neoplatymops como distinto de Molossops (ver más abajo).

Cambios taxonómicos al nivel de género. La diversidad taxonómica en esta categoría de acuerdo a Simmons (2005) alcanzaba 90 géneros en nueve familias. Desde entonces, tres nuevos nombres han sido introducidos, todos correspondientes a filostómidos nectarívoros: Xeronycteris vieirai, descrita a partir de ejemplares del noreste (Caatinga) de Brasil por Gregorin y Ditchfield (2005), Dryadonycteris capixaba, a partir de ejemplares del sureste (Bosque Atlántico) de Brasil por Nogueira et al. (2012), y Hsunycteris por Parlos et al. (2014) para tres especies previamente incluidas en Lonchophylla. En cuanto a sus relaciones, mientras que Hsunycteris es el clado hermano de los restantes Lonchophyllinae, dentro de estos, Xeronycteris está más cercanamente emparentado a Platalina; de otro lado, Dryadonycteris pertenece a la tribu Choeronycterina de los Glossophaginae.

Otros dos nombres genéricos que han sido validados como diferentes son los Stenodermatinos Vampyriscus Thomas, 1900, separado de Vampyressa (Hoofer y Baker 2006), y Dermanura Gervais, 1856, separado de Artibeus (Hoofer et al. 2008). Vampyressa (sensu lato) resultó polifilético, mientras que Dermanura constituyó el grupo hermano recíprocamente exclusivo de Artibeus (sensu stricto). Aunque no existe un patrón general, pues varía entre familias y subfamilias, la justificación para las distinciones genéricas incluye características cualitativas discretas. En el caso de Artibeus y Dermanura, ambos son distintos a nivel de tamaño, forma y detalles de la dentición (molares).

También validados como distintos son los géneros Neoplatymops Peterson, 1965 y Cabreramops Ibáñez, 1980, diferenciados morfológicamente de Molossops por Eger (2008). Además, el primero fue distinguido filogenéticamente por análisis de secuencias genéticas (Ammerman et al. 2012). La misma situación se presenta con los géneros Baeodon Miller, 1906, que fue separado de Rhogeessa, y Perimyotis Menu, 1984, separado de Pipistrellus, ambos con base en análisis filogenéticos de secuencias de ADN (Hoofer y Van Den Bussche 2003, Hoofer et al. 2006, Baird et al. 2008). Al comprobarse la polifilia del género Pipistrellus (Hoofer et al. 2006), se validó el nombre Perimyotis para incluir una única especie neotropical: P. subflavus. 
Finalmente, con base en un análisis filogenético molecular para casi todas las especies reconocidas en ese momento, Porter et al. (2007) describieron dos subgéneros dentro de Micronycteris: Leuconycteris (M. brosseti como única especie); y Schizonycteris (para M. minuta, M. sanborni y M. schmidtorum); los cuales se añadieron a los previamente existentes Micronycteris y Xenoctenes.

Otros estudios, tales como la revisión filogenética de Vespertilionidae (Hoofer y Van Den Bussche 2003), también aportaron cambios a la taxonomía de algunos géneros. Así, para Myotis se encontró que los subgéneros Leuconoe, Selyseus, y Myotis, no son monofiléticos. Sin embargo, las especies podían agruparse en dos clados correspondientes al Nuevo y Viejo Mundo. En esta división, el subgénero Myotis debe restringirse al Viejo Mundo, y para las especies Americanas se propuso Aeorestes Fitzinger, 1870. Sin embargo, Wilson (2008) indica que este nombre correspondería realmente a un sinónimo de Lasiurus debido a que la especie tipo A. villosissimus corresponde a un sinónimo de L. cinereus. Con respecto a Lasiurus, Hoofer y Van Den Bussche (2003) no distinguieron a Dasypterus como un género distinto, pero reconocen la necesidad de un mayor muestreo taxonómico. Quizás la propuesta más sorprendente fue la inclusión de Histiotus como un subgénero de Eptesicus, dada su estrecha relación con las especies Neotropicales de este último. Sin embargo, la parafilia de Eptesicus podría resolverse restringiendo este nombre a las especies del Neotrópico (E. fuscus es la especie tipo del género), y reconociendo a Histiotus así como a los grupos del Viejo Mundo como géneros distintos.

Dentro de Emballonuridae, la reciente descripción de una nueva especie de Peropteryx (Lim et al. 2010) permitió evaluar el soporte del subgénero Peronymus, concluyendo que este no tiene validez filogenética (ausencia de sinapomorfías). Finalmente, en Sturnira (Phyllostomidae) la validez del subgénero Corvira fue descartada al no hallarse soporte en los caracteres morfológicos ni en las relaciones filogenéticas respecto a otras especies del género (Velazco y Patterson 2013).

Cambios taxonómicos al nivel de especie. Esta es la categoría en la cual se ha producido el mayor número de cambios. Simmons (2005) incluyó 305 especies, mientras que nuestra revisión lleva este número a 380 para febrero de 2014. Ello significa un incremento promedio mayor a 8 especies/año (75 especies en 9 años), observándose una variación que va de 4 a 11 especies/año. Comparativamente, el incremento durante 1993-2005 fue de 191 especies a nivel mundial, a una tasa de 15.9 especies/año (Solari y Baker 2007).

El mayor incremento en número de especies tuvo lugar en la familia Phyllostomidae (Figura 1), con 46 especies en los últimos nueve años (más de 5 especies/año), superando las 1.58 especies/año del periodo 1993-2005 (ver Solari y Baker 2007). En cuanto a descripciones, la tendencia histórica mostrada por Wetterer et al. (2000) indica que el promedio fue de 0.92 especies/año durante 1900-1925, y 0.67 especies/año durante 1975-1993. Nuestros datos indican que para el periodo 2004-2013 este valor alcanza 3.55 especies/año (32 especies en nueve años).

Un total de 35 géneros en siete familias incorporan cambios a nivel de especie (Tabla 1). Mientras que algunos géneros incrementaron su diversidad en $100 \%$ o ligeramente menos (Anoura, Platyrrhinus, Lonchophylla), en la mayoría (12 géneros) el incremento 
ha sido de sólo una especie. Sin embargo, en géneros que han sido divididos, tales como Artibeus, Vampyressa o Molossops, el número de especies previamente incluidas ha disminuido. Tal como se ha reportado antes (Patterson 1996, 2000), no todo este incremento se refiere a nuevas especies propuestas recientemente. Así, del total de 75 especies adicionales, 30 (40\%) representan nombres previamente considerados

Figura 1. Distribución taxonómica (por familias) del total de cambios en el número de especies reconocidas en el periodo comprendido entre Simmons (2005) y esta revisión. Phyllostomidae = 63.53\%; Vespertilionidae $=12.94 \% ;$ Molossidae $=9.41 \% ;$ Natalidae $=$ $7.06 \%$; Mormoopidae $=$ $3.53 \%$; Thyropteridae $=$ $2.35 \%$; Emballonuridae $=$ $1.18 \%$.

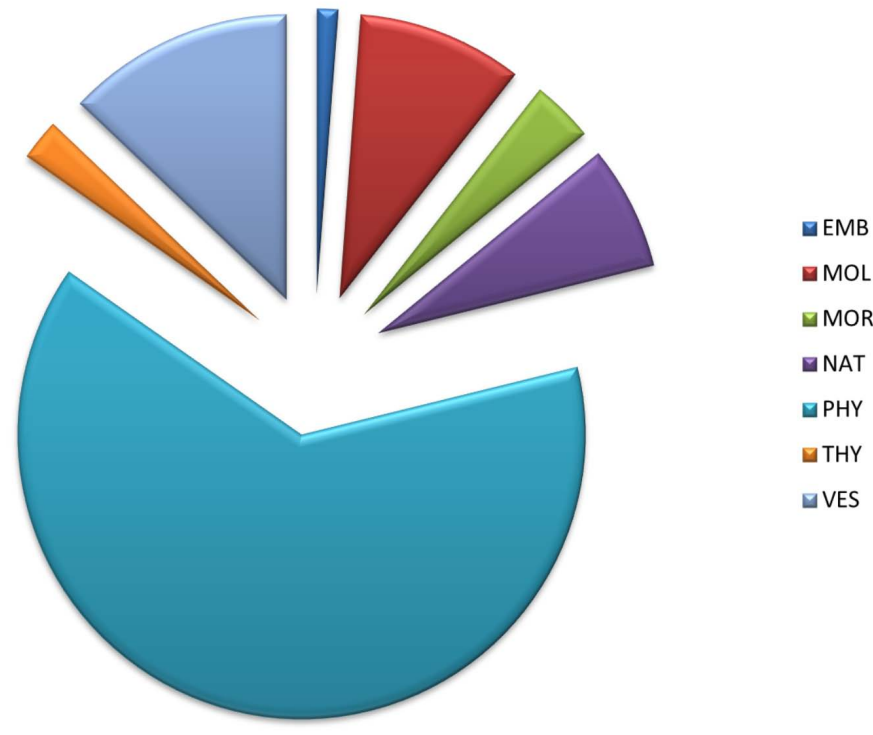

subespecies o sinónimos de especies válidas existentes.

Existen grupos particularmente complejos en los cuales ciertos nombres se describen o validan en una primera revisión taxonómica (e. g. Artibeus triomylus, Lophostoma aequatorialis, Natalus lanatus), pero en un trabajo posterior son sinonimizados con un nombre existente debido a que no se verifica su distinción real (ver Larsen et al. 2007; Velazco y Cadenillas 2011; López-Wilchis et al. 2012, respectivamente). En el caso particular de $L$. aequatorialis, este nuevo nombre se convierte en sinónimo de $L$. occidentalis, descrito en 1978, una vez que los tipos se comparan directamente usando datos morfológicos y moleculares. De manera opuesta, el nombre Sturnira ludovici se sinonimizó bajo S. oporaphilum por Gardner (2008) pero dicha decisión fue revertida en un análisis más completo del género (Velazco y Patterson 2013). Estos ejemplos muestran lo complicado que resulta tomar una decisión taxonómica, incluso cuando se dispone de información y análisis modernos, lo cual se refleja en variaciones entre años del número total de especies reconocidas.

Una evaluación de los criterios empleados para el reconocimiento de estas especies adicionales nos permite identificar dos criterios principales. El más empleado es el criterio de monofilia, es decir que cada entidad taxonómica (i. e. una especie o género) debe incluir sólo descendientes de un único ancestro. Para este criterio, es de interés encontrar evidencia aportada por análisis filogenéticos de datos morfológicos o moleculares que muestren que individuos asociados a un nombre de especie no forman un grupo único en la filogenia. Algunos ejemplos incluyen: a) la distinción de tres grupos a nivel de especie en lo que se anteriormente se denominaba Platyrrhinus dorsalis (sensu lato), con base en un análisis de caracteres morfológicos (Velazco 2005); y b) la división de Artibeus jamaicensis (sensu lato) en tres especies con base en el análisis filogenético de secuencias del citocromo b (Larsen et al. 2007). 
Un segundo criterio es el establecimiento de un grado suficiente de diferencia morfológica o morfométrica, es decir que el análisis estadístico de la variación dentro de una entidad taxonómica no revele diferencias significativas entre (sub)grupos. Se incluyen en este caso los análisis de tamaño y forma, que pueden incorporar pruebas multivariadas complejas como en la separación de Pteronotus paraguanensis respecto a P. parnellii (Gutiérrez y Molinari 2008), o una combinación de diferencias morfométricas y morfológicas discretas, como en la distinción entre Lonchophylla concava y L. mordax (Albuja y Gardner 2005).

\begin{tabular}{|c|c|c|c|c|c|}
\hline Género & $\begin{array}{c}\text { Especies sensu } \\
\text { Simmons (2005) }\end{array}$ & $\begin{array}{c}\text { Nuevas } \\
\text { descritas }\end{array}$ & $\begin{array}{c}\text { Nuevas } \\
\text { reconocidas }\end{array}$ & Sinonimizadas & $\begin{array}{l}\text { Especies } \\
\text { Actuales }\end{array}$ \\
\hline Peropteryx & 4 & 1 & 0 & 0 & 5 \\
\hline Pteronotus & 7 & 0 & 3 & 0 & 10 \\
\hline Anoura & 5 & 3 & 2 & 0 & 10 \\
\hline Dryadonycteris & $\mathbf{0}$ & 1 & 0 & 0 & 1 \\
\hline Hsunycteris $(*)$ & $\mathbf{0}$ & 0 & 3 & 0 & 3 \\
\hline Lichonycteris & 1 & 0 & 1 & 0 & 2 \\
\hline Lonchophylla $(*)$ & 7 & 7 & 1 & 3 & 12 \\
\hline Xeronycteris & $\mathbf{0}$ & 1 & 0 & 0 & 1 \\
\hline Lophostoma & 5 & 3 & 1 & 1 & 8 \\
\hline Micronycteris & 9 & 3 & 0 & 1 & 11 \\
\hline Carollia & 6 & 3 & 0 & 1 & 8 \\
\hline Sturnira & 14 & 3 & 5 & 2 & 20 \\
\hline Artibeus (s.s.) & 9 & 0 & 4 & 1 & 12 \\
\hline Dermanura (s.s.) & 9 & 0 & 3 & 1 & 11 \\
\hline Chiroderma & 5 & 1 & 0 & 0 & 6 \\
\hline Platyrrhinus & 10 & 7 & 3 & 0 & 20 \\
\hline Vampyressa $(*)$ & 6 & 0 & 0 & 3 & 3 \\
\hline Vampyriscus $(*)$ & $\mathbf{0}$ & 0 & 3 & 0 & 3 \\
\hline Vampyrodes & 1 & 0 & 1 & 0 & 2 \\
\hline Thyroptera & 3 & 2 & 0 & 0 & 5 \\
\hline Chilonatalus & 2 & 0 & 1 & 0 & 3 \\
\hline Natalus & 5 & 1 & 4 & 3 & 7 \\
\hline Cabreramops $(*)$ & $\mathbf{0}$ & 0 & 1 & 0 & 1 \\
\hline Cynomops & 5 & 0 & 1 & 0 & 6 \\
\hline Eumops & 10 & 1 & 3 & 0 & 14 \\
\hline Molossops $(*)$ & 4 & 0 & 0 & 2 & 2 \\
\hline Molossus & 8 & 1 & 1 & 0 & 10 \\
\hline Neoplatymops $(*)$ & $\mathbf{0}$ & 0 & 1 & 0 & 1 \\
\hline Promops & 2 & 0 & 1 & 0 & 3 \\
\hline Eptesicus & 8 & 1 & 0 & 0 & 9 \\
\hline Baeodon $(*)$ & $\mathbf{0}$ & 0 & 2 & 0 & 2 \\
\hline Myotis & 20 & 4 & 3 & 0 & 27 \\
\hline Perimyotis $(* *)$ & $\mathbf{0}$ & 0 & 1 & 0 & 1 \\
\hline Pipistrellus (**) & 1 & 0 & 0 & 1 & $\mathbf{0}$ \\
\hline \multirow[t]{2}{*}{ Rhogeessa $(*)$} & 10 & 2 & 1 & 2 & 11 \\
\hline & 177 & 45 & 50 & 21 & 250 \\
\hline
\end{tabular}

Tabla 1. Listado de los 35 géneros actuales en los cuales se presentan cambios taxonómicos resultantes en incrementos en el número de especies reconocidas respecto al número presentado por Simmons (2005). $\left({ }^{*}\right)$ Casos particulares en
los que un número de
especies cambian entre
géneros, usualmente al
validar uno de ellos como
distinto. Este cambio no
implica incrementos o
disminuciones en la di-
versidad total, ni en el
número de nuevas espe-
cies; se cuentan como
nuevas reconocidas (en el
"nuevo" género) y como
sinonimizadas (en el gé-
nero original).
(**) Pipistrellus solo in-
cluía una especie Neo-
tropical; al comprobarse
su polifilia (Hoofer et al.
2006 ), se validó el nom-
bre Perimyotis para esta
única especie P. subfla-
vus. 
Otros criterios menos comunes para reconocer especies de murciélagos Neotropicales han sido empleados en combinación con los criterios previamente mencionados. Por ejemplo, disyunción geográfica entre poblaciones, asociada a la presencia de caracteres discretos distintivos, como en el caso de Lichonycteris obscura y L. degener (Griffiths y Gardner 2008), o Molossus bondae y M. currentium (Eger 2008). Igualmente, la presencia de distintos cariotipos como evidencia de aislamiento reproductivo y genético, como en el caso de Eumops wilsoni respecto a E. glaucinus y E. floridanus (Baker et al. 2009).

Figura 2. Aporte de nuevas especies descritas (porción azul), nuevas especies reconocidas como válidas (en verde), y nombres sinonimizados (en rojo) al número acumulado de cambios específicos por año en murciélagos Neotropicales.

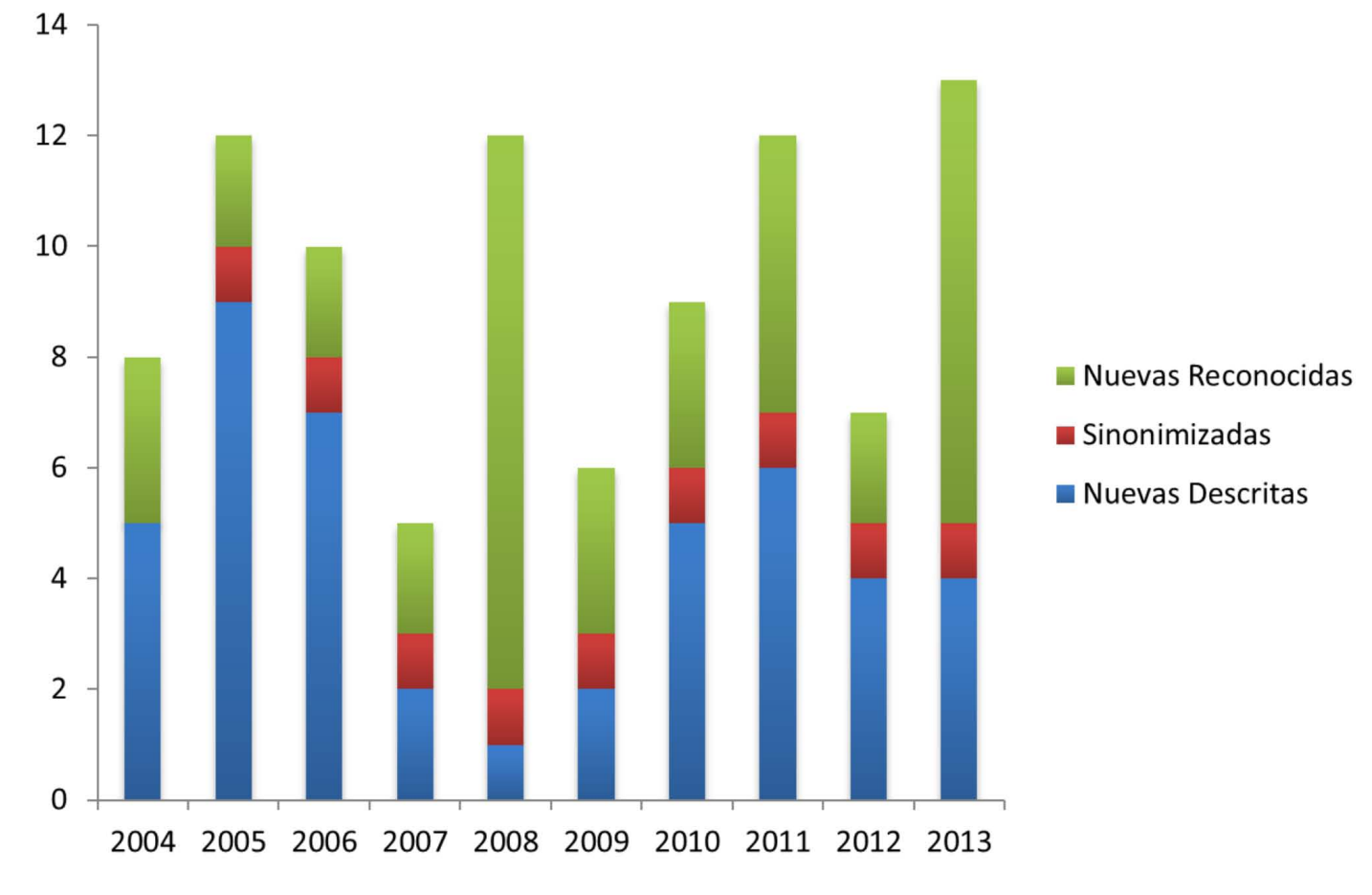

Aunque nuestra revisión revela una diversidad de aproximaciones a la resolución taxonómica de los límites entre especies de murciélagos, no deja de ser sorprendente la tasa de incremento en el número de especies reconocidas. Ceballos y Ehrlich (2009) encontraron que el incremento global en la riqueza de especies de murciélagos fue mayor al esperado dentro de los mamíferos, considerando la diversidad del orden. En los nueve años transcurridos desde el cierre de la tercera edición del listado de mamíferos del mundo (Wilson y Reeder 2005), se ha pasado de 305 a 380 especies de murciélagos Neotropicales, lo cual representa un incremento aproximado de $24 \%$ respecto al total original (Figura 3). De este incremento, 45 especies (60\%; Tabla 1) son especies nuevas, previamente desconocidas. Poco menos de la mitad de ellas (20) corresponden a especies crípticas diferenciadas gracias a avances metodológicos.

\section{Discusión}

El resultado más importante de esta revisión es una lista actualizada de las especies Neotropicales de murciélagos, que incluye 380 especies en 98 géneros (Tabla 2). Obviamente, ésta no pretende ser una lista definitiva, e incluye decisiones nomenclaturales a discreción de los autores que no implican un juicio sobre la validez de algún trabajo en particular. En adición a estas 380 especies, otras cinco han sido identificadas pero no nombradas; una en Carollia (Solari y Baker 2006), tres en Sturnira 
(Velazco y Patterson 2013), y una en Hsunycteris (Parlos et al. 2014). Igualmente, podría tenerse en cuenta las especies consideradas extintas por Simmons (2005): Mormoops magna, Pteronotus pristinus, y Phyllonycteris major, o por la UICN (Unión Internacional para la Conservación de la Naturaleza): Desmodus draculae.

\begin{tabular}{|c|c|c|c|}
\hline Género & Especie & Autor y año & Referencias \\
\hline \multicolumn{4}{|c|}{ Familia Emballonuridae } \\
\hline Balantiopteryx & infusca & (Thomas, 1897) & \\
\hline Balantiopteryx & io & Thomas, 1904 & \\
\hline Balantiopteryx & plicata & W. Peters, 1867 & \\
\hline Centronycteris & centralis & Thomas, 1912 & \\
\hline Centronycteris & maximiliani & (Fischer, 1829) & \\
\hline Cormura & brevirostris & (Wagner, 1843) & \\
\hline Cyttarops & alecto & Thomas, 1913 & \\
\hline Diclidurus & albus & Wied-Neuwied, 1820 & \\
\hline Diclidurus & ingens & Hernández-Camacho, 1955 & \\
\hline Diclidurus & isabellus & (Thomas, 1920) & \\
\hline Diclidurus & scutatus & W. Peters, 1869 & \\
\hline Peropteryx & kappleri & W. Peters, 1867 & \\
\hline Peropteryx & leucoptera & W. Peters, 1867 & 60 \\
\hline Peropteryx & macrotis & (Wagner, 1843) & \\
\hline Peropteryx & pallidoptera & Lim et al., 2010 & 60 \\
\hline Peropteryx & trinitatis & Miller, 1899 & \\
\hline Rhynchonycteris & naso & (Wied-Neuwied, 1820) & \\
\hline Saccopteryx & antioquensis & Muñoz \& Cuartas-Calle, 2001 & \\
\hline Saccopteryx & bilineata & (Temminck, 1838) & \\
\hline Saccopteryx & canescens & Thomas, 1901 & \\
\hline Saccopteryx & gymnura & Thomas, 1901 & \\
\hline Saccopteryx & leptura & (Schreber, 1774) & \\
\hline \multicolumn{4}{|c|}{ Familia Noctilionidae } \\
\hline Noctilio & albiventris & Desmarest, 1818 & \\
\hline Noctilio & leporinus & (Linnaeus, 1758) & \\
\hline \multicolumn{4}{|c|}{ Familia Mormoopidae } \\
\hline Mormoops & blainvillei & Leach, 1821 & \\
\hline Mormoops & megalophylla & (W. Peters, 1864) & \\
\hline Pteronotus & davyi & Gray, 1838 & \\
\hline Pteronotus & gymnonotus & (J. A. Wagner, 1843) & \\
\hline Pteronotus & macleayii & (Gray, 1839) & \\
\hline Pteronotus & mesoamericanus & Smith, 1972 & 14,20 \\
\hline Pteronotus & paraguanensis & Linares \& Ojasti, 1974 & 41 \\
\hline Pteronotus & parnellii & (Gray, 1843) & $14,20,41$ \\
\hline Pteronotus & personatus & (J. A. Wagner, 1843) & \\
\hline Pteronotus & quadridens & (Gundlach, 1840) & \\
\hline Pteronotus & rubiginosus & (J. A. Wagner, 1843) & 20,41 \\
\hline
\end{tabular}

Tabla 2. Lista actualizada, hasta el 15 de febrero de 2014, de los géneros y especies de murciélagos Neotropicales. Sólo se incluyen subfamilias para los Phyllostomidae, siguiendo el esquema de Baker et al. (2003). Las referencias (ver códigos en la Bibliografía) son sólo para aquellas especies cuyo estado taxonómico ha cambiado en el periodo evaluado por esta revisión. 


\begin{tabular}{|c|c|c|c|}
\hline Género & Especie & Autor y año & Referencias \\
\hline \multicolumn{4}{|c|}{ Familia Phyllostomidae } \\
\hline \multicolumn{3}{|c|}{ Subfamilia Macrotinae } & 8 \\
\hline Macrotus & waterhousii & Gray, 1843 & \\
\hline \multicolumn{3}{|c|}{ Subfamilia Micronycterinae } & 8 \\
\hline Lampronycteris & brachyotis & (Dobson, 1879) & \\
\hline Micronycteris & brosseti & Simmons \& Voss, 1998 & \\
\hline Micronycteris & buriri & Larsen et al., 2011 & 56 \\
\hline Micronycteris & giovanniae & Baker \& Fonseca, 2007 & 30 \\
\hline Micronycteris & hirsuta & (W. Peters, 1869) & \\
\hline Micronycteris & matses & Simmons et al., 2002 & \\
\hline Micronycteris & megalotis & (Gray, 1842) & 56,83 \\
\hline Micronycteris & microtis & Miller, 1898 & 83 \\
\hline Micronycteris & minuta & (P. Gervais, 1856) & 75 \\
\hline Micronycteris & sanborni & Simmons, 1996 & \\
\hline Micronycteris & schmidtorum & (Sanborn, 1935) & \\
\hline Micronycteris & yatesi & Siles et al. 2013 & 86 \\
\hline \multicolumn{4}{|c|}{ Subfamilia Desmodontinae } \\
\hline Desmodus & rotundus & (É. Geoffroy St.-Hilaire, 1810) & \\
\hline Diaemus & youngi & (Jentink, 1893) & \\
\hline Diphylla & ecaudata & Spix, 1823 & \\
\hline \multicolumn{3}{|c|}{ Subfamilia Lonchorhininae } & 8 \\
\hline Lonchorhina & aurita & Tomes, 1863 & \\
\hline Lonchorhina & fernandezi & Ochoa \& Ibáñez, 1984 & \\
\hline Lonchorhina & inusitata & Handley \& Ochoa, 1997 & \\
\hline Lonchorhina & marinkellei & $\begin{array}{l}\text { Hernández-Camacho \& } \\
\text { Cadena, } 1978\end{array}$ & \\
\hline Lonchorhina & orinocensis & Linares \& Ojasti, 1971 & \\
\hline \multicolumn{3}{|c|}{ Subfamilia Phyllostominae } & 8 \\
\hline Chrotopterus & auritus & (W. Peters, 1856) & \\
\hline Lophostoma & brasiliense & W. Peters, 1867 & \\
\hline Lophostoma & carrikeri & (J. A. Allen, 1910) & \\
\hline Lophostoma & evotis & (Davis \& Carter, 1978) & \\
\hline Lophostoma & kalkoae & Velazco \& Gardner, 2012 & 109 \\
\hline Lophostoma & occidentalis & (Davis \& Carter, 1978) & 9,107 \\
\hline Lophostoma & schulzi & (Genoways \& Williams, 1980) & \\
\hline Lophostoma & silvicolum & d'Orbigny, 1836 & 9,107 \\
\hline Lophostoma & yasuni & Fonseca \& Pinto, 2004 & 29 \\
\hline Macrophyllum & macrophyllum & (Schinz, 1821) & \\
\hline Mimon & bennettii & (Gray, 1838) & \\
\hline Mimon & cozumelae & Goldman, 1914 & \\
\hline Mimon & crenulatum & (É. Geoffroy St.-Hilaire, 1803) & \\
\hline Mimon & koepckeae & Gardner \& Patton, 1972 & \\
\hline Phylloderma & stenops & W. Peters, 1865 & \\
\hline Phyllostomus & discolor & (Wagner, 1843) & \\
\hline
\end{tabular}




\begin{tabular}{|c|c|c|c|}
\hline Género & Especie & Autor y año & Referencias \\
\hline Phyllostomus & elongatus & (É. Geoffroy St.-Hilaire, 1810) & \\
\hline Phyllostomus & hastatus & (Pallas, 1767) & \\
\hline Phyllostomus & latifolius & (Thomas, 1901) & \\
\hline Tonatia & bidens & (Spix, 1823) & \\
\hline Tonatia & saurophila & Koopman \& Williams, 1951 & \\
\hline Trachops & cirrhosus & (Spix, 1823) & \\
\hline Vampyrum & spectrum & (Linnaeus, 1758) & \\
\hline \multicolumn{2}{|c|}{ Subfamilia Glossophaginae } & & 8 \\
\hline Anoura & aequatoris & (Lönnberg, 1921) & 64 \\
\hline Anoura & cadenai & Mantilla-Meluk \& Baker, 2006 & 64 \\
\hline Anoura & carishina & Mantilla-Meluk \& Baker, 2010 & 65 \\
\hline Anoura & caudifer & (É. Geoffroy St.-Hilaire, 1818) & 64 \\
\hline Anoura & cultrata & Handley, 1960 & \\
\hline Anoura & fistulata & Muchhala et al., 2005 & 72 \\
\hline Anoura & geoffroyi & Gray, 1838 & 65 \\
\hline Anoura & latidens & Handley, 1984 & \\
\hline Anoura & luismanueli & Molinari, 1994 & \\
\hline Anoura & peruana & (Tschudi, 1844) & 65 \\
\hline Brachyphylla & cavernarum & Gray, 1834 & \\
\hline Brachyphylla & nana & Miller, 1902 & \\
\hline Choeroniscus & godmani & (Thomas, 1903) & \\
\hline Choeroniscus & minor & (Peters, 1868) & \\
\hline Choeroniscus & periosus & Handley, 1966 & \\
\hline Choeronycteris & mexicana & Tschudi, 1844 & \\
\hline Dryadonycteris & capixaba & Nogueira et al., 2012 & 74 \\
\hline Erophylla & bombifrons & (Miller, 1899) & \\
\hline Erophylla & sezekorni & (Gundlach, 1861) & \\
\hline Glossophaga & commissarisi & Gardner, 1962 & \\
\hline Glossophaga & leachii & Gray, 1844 & \\
\hline Glossophaga & longirostris & Miller, 1898 & \\
\hline Glossophaga & morenoi & Martínez and Villa-R., 1938 & \\
\hline Glossophaga & soricina & (Pallas, 1766) & \\
\hline Hylonycteris & underwoodi & Thomas, 1903 & \\
\hline Leptonycteris & curasoae & Miller, 1900 & \\
\hline Leptonycteris & nivalis & (Saussure, 1860) & \\
\hline Leptonycteris & yerbabuenae & Martínez \& Villa-R., 1940 & \\
\hline Lichonycteris & degener & Miller, 1931 & 38 \\
\hline Lichonycteris & obscura & Thomas, 1895 & 38 \\
\hline Monophyllus & plethodon & Miller, 1900 & \\
\hline Monophyllus & redmani & Leach, 1821 & \\
\hline Musonycteris & harrisoni & Schaldach \& McLaughlin, 1960 & \\
\hline Phyllonycteris & poeyi & Gundlach, 1861 & \\
\hline Phyllonycteris & aphylla & (Miller, 1898) & \\
\hline Scleronycteris & ega & Thomas, 1912 & \\
\hline
\end{tabular}

continúa... 


\begin{tabular}{|c|c|c|c|}
\hline Género & Especie & Autor y año & Referencias \\
\hline \multicolumn{3}{|c|}{ Subfamilia Lonchophyllinae } & 8 \\
\hline Hsunycteris & cadenai & (Woodman \& Timm, 2006) & 80,123 \\
\hline Hsunycteris & pattoni & (Woodman \& Timm, 2006) & 80,123 \\
\hline Hsunycteris & thomasi & (J. A. Allen, 1904) & 80,123 \\
\hline Lionycteris & spurrelli & Thomas, 1913 & \\
\hline Lonchophylla & bokermanni & Sazima et al., 1978 & 26 \\
\hline Lonchophylla & chocoana & Dávalos, 2004 & 18 \\
\hline Lonchophylla & concava & Goldman, 1914 & 2,122 \\
\hline Lonchophylla & dekeyseri & Taddei et al., 1983 & \\
\hline Lonchophylla & fornicata & Woodman, 2007 & 122 \\
\hline Lonchophylla & handleyi & Hill, 1980 & \\
\hline Lonchophylla & hesperia & G. M. Allen, 1908 & \\
\hline Lonchophylla & mordax & Thomas, 1903 & 2 \\
\hline Lonchophylla & orcesi & Albuja \& Gardner, 2004 & 2 \\
\hline Lonchophylla & orienticollina & Dávalos \& Corthals, 2008 & 21 \\
\hline Lonchophylla & perachii & Dias et al., 2013 & 26 \\
\hline Lonchophylla & robusta & Miller, 1912 & \\
\hline Platalina & genovensium & Thomas, 1928 & \\
\hline Xeronycteris & vieirai & Gregorin \& Ditchfield, 2005 & 34 \\
\hline \multicolumn{3}{|c|}{ Subfamilia Carolliinae } & 8 \\
\hline Carollia & benkeithi & Solari \& Baker, 2006 & 88 \\
\hline Carollia & brevicauda & (Schinz, 1821) & 16,125 \\
\hline Carollia & castanea & H. Allen, 1890 & 88 \\
\hline Carollia & manu & Pacheco et al., 2004 & 77 \\
\hline Carollia & monohernandezi & Muñoz et al., 2004 & 73,125 \\
\hline Carollia & perspicillata & (Linnaeus, 1758) & 73 \\
\hline Carollia & sowelli & Baker et al., 2002 & \\
\hline Carollia & subrufa & (Hahn, 1905) & \\
\hline \multicolumn{3}{|c|}{ Subfamilia "Glyphonycterinae" (A) } & 8 \\
\hline Glyphonycteris & behnii & (W. Peters, 1865) & \\
\hline Glyphonycteris & daviesi & (Hill, 1965) & \\
\hline Glyphonycteris & sylvestris & (Thomas, 1896) & \\
\hline Neonycteris & pusilla & (Sanborn, 1949) & \\
\hline Trinycteris & nicefori & (Sanborn, 1949) & \\
\hline \multicolumn{3}{|c|}{ Subfamilia "Rhinophyllinae" (A) } & 8 \\
\hline Rhinophylla & alethina & Handley, 1966 & \\
\hline Rhinophylla & fischerae & Carter, 1966 & \\
\hline Rhinophylla & pumilio & W. Peters, 1865 & \\
\hline \multicolumn{4}{|c|}{ Subfamilia Stenodermatinae } \\
\hline Ametrida & centurio & Gray, 1847 & \\
\hline Ardops & nichollsi & (Thomas, 1891) & \\
\hline Ariteus & flavescens & (Gray, 1831) & \\
\hline Artibeus & aequatorialis & K. Andersen, 1906 & 55 \\
\hline Artibeus & amplus & Handley, 1987 & \\
\hline
\end{tabular}




\begin{tabular}{|c|c|c|c|}
\hline Género & Especie & Autor y año & Referencias \\
\hline Artibeus & concolor & W. Peters, 1865 & 46 \\
\hline Artibeus & fimbriatus & Gray, 1838 & \\
\hline Artibeus & fraterculus & Anthony, 1924 & \\
\hline Artibeus & hirsutus & W. Andersen, 1906 & \\
\hline Artibeus & inopinatus & Davis \& Carter, 1964 & \\
\hline Artibeus & jamaicensis & Leach, 1821 & 54,55 \\
\hline Artibeus & lituratus & (Olfers, 1818) & 46 \\
\hline Artibeus & obscurus & (Schinz, 1821) & \\
\hline Artibeus & planirostris & (Spix, 1823) & 54 \\
\hline Artibeus & schwartzi & Jones, 1978 & 54 \\
\hline Centurio & senex & Gray, 1842 & \\
\hline Chiroderma & doriae & Thomas, 1891 & \\
\hline Chiroderma & improvisum & Baker \& Genoways, 1976 & \\
\hline Chiroderma & salvini & Dobson, 1878 & \\
\hline Chiroderma & trinitatum & Goodwin, 1958 & \\
\hline Chiroderma & villosum & W. Peters, 1860 & \\
\hline Chiroderma & vizzotoi & Taddei \& Lim, 2010 & 94 \\
\hline Dermanura & anderseni & (Osgood, 1916) & 46,90 \\
\hline Dermanura & azteca & (K. Andersen, 1906) & 46,90 \\
\hline Dermanura & bogotensis & (K. Andersen, 1906) & $46,58,90$ \\
\hline Dermanura & cinerea & P. Gervais, 1856 & 46,90 \\
\hline Dermanura & glauca & (Thomas, 1893) & $46,58,90$ \\
\hline Dermanura & gnoma & (Handley, 1987) & 46,90 \\
\hline Dermanura & phaeotis & Miller, 1902 & 46,90 \\
\hline Dermanura & rava & Miller, 1902 & 46,90 \\
\hline Dermanura & rosenbergi & Thomas, 1897 & 46,90 \\
\hline Dermanura & tolteca & (Saussure, 1860) & 46,90 \\
\hline Dermanura & watsoni & Thomas, 1901 & 46,90 \\
\hline Ectophylla & alba & H. Allen, 1892 & \\
\hline Enchisthenes & hartii & (Thomas, 1892) & \\
\hline Mesophylla & macconnelli & Thomas, 1901 & 44 \\
\hline Phyllops & falcatus & W. Peters, 1865 & \\
\hline Platyrrhinus & albericoi & Velazco, 2005 & 106 \\
\hline Platyrrhinus & angustirostris & Velazco et al., 2010 & 114 \\
\hline Platyrrhinus & aquilus & (Handley \& Ferris, 1972) & 108 \\
\hline Platyrrhinus & aurarius & (Handley \& Ferris, 1972) & \\
\hline Platyrrhinus & brachycephalus & (Rouk \& Carter, 1972) & \\
\hline Platyrrhinus & chocoensis & Alberico \& Velasco-A., 1991 & \\
\hline Platyrrhinus & dorsalis & (Thomas, 1900) & $\begin{array}{l}106,108, \\
113\end{array}$ \\
\hline Platyrrhinus & fusciventris & Velazco et al., 2010 & 114 \\
\hline Platyrrhinus & helleri & (W. Peters, 1866) & $\begin{array}{l}\text { 106, 110, } \\
114\end{array}$ \\
\hline Platyrrhinus & incarum & (Thomas, 1912) & 110 \\
\hline Platyrrhinus & infuscus & (W. Peters, 1880) & \\
\hline
\end{tabular}




\begin{tabular}{|c|c|c|c|}
\hline Género & Especie & Autor y año & Referencias \\
\hline Platyrrhinus & ismaeli & Velazco, 2005 & 106 \\
\hline Platyrrhinus & lineatus & (É. Geoffroy St.-Hilaire, 1810) & 113 \\
\hline Platyrrhinus & masu & Velazco, 2005 & 106 \\
\hline Platyrrhinus & matapalensis & Velazco, 2005 & 106 \\
\hline Platyrrhinus & nigellus & (Gardner \& Carter, 1972) & 113 \\
\hline Platyrrhinus & nitelinea & Velazco \& Gardner, 2009 & 108 \\
\hline Platyrrhinus & recifinus & (Thomas, 1901) & \\
\hline Platyrrhinus & umbratus & (Lyon, 1902) & 108 \\
\hline Platyrrhinus & vittatus & (W. Peters, 1859) & 106 \\
\hline Pygoderma & bilabiatum & (J. A. Wagner, 1843) & \\
\hline Sphaeronycteris & toxophyllum & W. Peters, 1882 & \\
\hline Stenoderma & rufum & Desmarest, 1820 & \\
\hline Uroderma & bilobatum & W. Peters, 1866 & \\
\hline Uroderma & magnirostrum & Davis, 1968 & \\
\hline Vampyressa & melissa & Thomas, 1926 & 44 \\
\hline Vampyressa & pusilla & (J. A. Wagner, 1843) & 44 \\
\hline Vampyressa & thyone & Thomas, 1909 & 44 \\
\hline Vampyriscus & bidens & (Dobson, 1878) & 44 \\
\hline Vampyriscus & brocki & (Peterson, 1968) & 44 \\
\hline Vampyriscus & nymphaea & (Thomas, 1909) & 44 \\
\hline Vampyrodes & caraccioli & (Thomas, 1889) & 112 \\
\hline Vampyrodes & major & G. M. Allen, 1908 & 112 \\
\hline Sturnira & angeli & de la Torre, 1961 & 111 \\
\hline Sturnira & aratathomasi & Peterson \& Tamsitt, 1968 & \\
\hline Sturnira & bidens & (Thomas, 1915) & \\
\hline Sturnira & bogotensis & Shamel, 1927 & \\
\hline Sturnira & erythromos & (Tschudi, 1844) & \\
\hline Sturnira & hondurensis & Goodwin, 1940 & 32 \\
\hline Sturnira & koopmanhilli & McCarthy et al., 2006 & 66 \\
\hline Sturnira & lilium & (É. Geoffroy St.-Hilaire, 1810) & 111 \\
\hline Sturnira & ludovici & Anthony, 1924 & 32,111 \\
\hline Sturnira & luisi & Davis, 1980 & 111 \\
\hline Sturnira & magna & de la Torre, 1966 & \\
\hline Sturnira & mistratensis & $\begin{array}{l}\text { Contreras-Vega \& Cadena, } \\
2000\end{array}$ & \\
\hline Sturnira & mordax & (Goodwin, 1938) & \\
\hline Sturnira & nana & Gardner \& O'Neill, 1971 & \\
\hline Sturnira & oporaphilum & (Tschudi, 1844) & 32,111 \\
\hline Sturnira & parvidens & Goldman, 1917 & 111 \\
\hline Sturnira & paulsoni & de la Torre \& Schwartz, 1966 & 111 \\
\hline Sturnira & perla & Jarrín \& Kunz, 2011 & 48 \\
\hline Sturnira & sorianoi & $\begin{array}{l}\text { Sánchez-Hernández et al., } \\
2005\end{array}$ & 85 \\
\hline Sturnira & tildae & de la Torre, 1959 & \\
\hline
\end{tabular}

continúa... 


\begin{tabular}{|c|c|c|c|}
\hline Género & Especie & Autor y año & Referencias \\
\hline \multicolumn{4}{|l|}{ Familia Natalidae } \\
\hline Chilonatalus & macer & Miller, 1914 & 100 \\
\hline Chilonatalus & micropus & (Dobson, 1880) & 100 \\
\hline Chilonatalus & tumidifrons & Miller, 1903 & 100 \\
\hline Natalus & jamaicensis & Goodwin, 1959 & 19,100 \\
\hline Natalus & macrourus & P. Gervais, 1856 & 31,100 \\
\hline Natalus & major & Miller, 1902 & 100 \\
\hline Natalus & mexicanus & Miller, 1902 & $\begin{array}{l}19,62,98 \\
100\end{array}$ \\
\hline Natalus & primus & Anthony, 1919 & 100 \\
\hline Natalus & stramineus & Gray, 1838 & $\begin{array}{l}19,98,99 \\
100\end{array}$ \\
\hline Natalus & tumidirostris & Miller, 1900 & 99,100 \\
\hline Nyctiellus & lepidus & (Gervais, 1837) & 100 \\
\hline \multicolumn{4}{|c|}{ Familia Furipteridae } \\
\hline Amorphochilus & schnablii & W. Peters, 1877 & \\
\hline Furipterus & horrens & (F. Cuvier, 1828) & \\
\hline \multicolumn{4}{|c|}{ Familia Thyropteridae } \\
\hline Thyroptera & devivoi & Gregorin et al., 2006 & 36 \\
\hline Thyroptera & discifera & (Lichtenstein \& W. Peters, 1854) & \\
\hline Thyroptera & lavali & Pine, 1993 & \\
\hline Thyroptera & tricolor & Spix, 1823 & \\
\hline Thyroptera & wynneae & Velazco et al., 2014 & 115 \\
\hline \multicolumn{4}{|c|}{ Familia Molossidae } \\
\hline Cabreramops & aequatorianus & (Cabrera, 1917) & 28 \\
\hline Cynomops & abrasus & (Temminck, 1826) & \\
\hline Cynomops & greenhalli & Goodwin, 1958 & \\
\hline Cynomops & mexicanus & Jones \& Genoways, 1967 & \\
\hline Cynomops & milleri & (Osgood, 1914) & 28 \\
\hline Cynomops & paranus & (Thomas, 1901) & \\
\hline Cynomops & planirostris & (W. Peters, 1866) & \\
\hline Eumops & auripendulus & (G. Shaw, 1800) & \\
\hline Eumops & bonariensis & (W. Peters, 1874) & 28 \\
\hline Eumops & dabbenei & Thomas, 1914 & \\
\hline Eumops & delticus & Thomas, 1923 & 28 \\
\hline Eumops & floridanus & G. M. Allen, 1932 & 101 \\
\hline Eumops & glaucinus & (J. A. Wagner, 1843) & 10,101 \\
\hline Eumops & hansae & Sanborn, 1932 & \\
\hline Eumops & maurus & (Thomas, 1901) & \\
\hline Eumops & nanus & (Miller, 1900) & 28 \\
\hline Eumops & patagonicus & Thomas, 1924 & \\
\hline Eumops & perotis & (Schinz, 1821) & \\
\hline Eumops & trumbulli & (Thomas, 1901) & \\
\hline Eumops & underwoodi & Goodwin, 1940 & \\
\hline Eumops & wilsoni & Baker et al., 2009 & 10 \\
\hline
\end{tabular}




\begin{tabular}{|c|c|c|c|}
\hline Género & Especie & Autor y año & Referencias \\
\hline Molossops & neglectus & Williams \& Genoways, 1980 & 3 \\
\hline Molossops & temminckii & (Burmeister, 1854) & 3 \\
\hline Molossus & alvarezi & González-Ruiz et al., 2011 & 33 \\
\hline Molossus & aztecus & Saussure, 1860 & \\
\hline Molossus & barnesi & Thomas, 1905 & \\
\hline Molossus & bondae & J. A. Allen, 1904 & 28,61 \\
\hline Molossus & coibensis & J. A. Allen, 1904 & \\
\hline Molossus & currentium & Thomas, 1901 & 28,61 \\
\hline Molossus & molossus & (Pallas, 1766) & \\
\hline Molossus & pretiosus & Miller, 1902 & \\
\hline Molossus & rufus & É. Geoffroy St.-Hilaire, 1805 & \\
\hline Molossus & sinaloae & J. A. Allen, 1906 & 33 \\
\hline Mormopterus & kalinowskii & (Thomas, 1893) & \\
\hline Mormopterus & minutus & (Miller, 1899) & \\
\hline Mormopterus & phrudus & Handley, 1956 & \\
\hline Neoplatymops & mattogrossensis & (Vieira, 1942) & 3,28 \\
\hline Nyctinomops & aurispinosus & (T. R. Peale, 1848) & \\
\hline Nyctinomops & femorosaccus & (Merriam, 1889) & \\
\hline Nyctinomops & laticaudatus & (É. Geoffroy St.-Hilaire, 1805) & \\
\hline Nyctinomops & macrotis & (Gray, 1839) & \\
\hline Promops & centralis & Thomas, 1915 & 35 \\
\hline Promops & davisoni & Thomas, 1921 & 35 \\
\hline Promops & nasutus & (Spix, 1823) & \\
\hline Tadarida & brasiliensis & (I. Geoffroy St.-Hilaire, 1824) & \\
\hline Tomopeas & ravus & Miller, 1900 & \\
\hline \multicolumn{4}{|c|}{ Familia Vespertilionidae } \\
\hline Baeodon & alleni & (Thomas, 1892) & $4,5,43$ \\
\hline Baeodon & gracilis & (Miller, 1897) & $4,5,43$ \\
\hline Bauerus & dubiaquercus & (Van Gelder, 1959) & \\
\hline Corynorhinus & towsendii & (Cooper, 1837) & \\
\hline Eptesicus & andinus & J. A. Allen, 1914 & \\
\hline Eptesicus & brasiliensis & (Desmarest, 1819) & \\
\hline Eptesicus & chiriquinus & Thomas, 1920 & \\
\hline Eptesicus & diminutus & Osgood, 1915 & \\
\hline Eptesicus & furinalis & (d'Orbigny \& Gervais, 1847) & \\
\hline Eptesicus & fuscus & (Palisot de Beauvois, 1796) & \\
\hline Eptesicus & guadeloupensis & Genoways \& Baker, 1975 & \\
\hline Eptesicus & innoxius & (P. Gervais, 1841) & \\
\hline Eptesicus & taddeii & Miranda et al., 2006 & 67 \\
\hline Histiotus & alienus & Thomas, 1916 & 42,43 \\
\hline Histiotus & humboldti & Handley, 1996 & 42,43 \\
\hline Histiotus & laephotis & Thomas, 1916 & 42,43 \\
\hline Histiotus & macrotus & (Poeppig, 1835) & 42,43 \\
\hline Histiotus & magellanicus & (R. A. Philippi, 1866) & 42,43 \\
\hline
\end{tabular}




\begin{tabular}{|c|c|c|c|}
\hline Género & Especie & Autor y año & Referencias \\
\hline Histiotus & montanus & $\begin{array}{l}\text { (R. A. Philippi \& Landbeck, } \\
\text { 1861) }\end{array}$ & 42,43 \\
\hline Histiotus & velatus & (I. Geoffroy St.-Hilaire, 1824) & 42,43 \\
\hline Lasiurus & atratus & Handley, 1996 & \\
\hline Lasiurus & blossevillii & (Lesson, 1826) & \\
\hline Lasiurus & castaneus & Handley, 1960 & \\
\hline Lasiurus & cinereus & (Palisot de Beauvois, 1796) & \\
\hline Lasiurus & degelidus & Miller, 1931 & \\
\hline Lasiurus & ebenus & Fazzolari-Correa, 1994 & \\
\hline Lasiurus & ega & (P. Gervais, 1856) & \\
\hline Lasiurus & egregius & (W. Peters, 1870) & \\
\hline Lasiurus & insularis & Hall \& Jones, 1961 & \\
\hline Lasiurus & intermedius & H. Allen, 1862 & \\
\hline Lasiurus & minor & Miller, 1931 & \\
\hline Lasiurus & pfeifferi & (Gundlach, 1861) & \\
\hline Lasiurus & salinae & Thomas, 1902 & \\
\hline Lasiurus & varius & Poeppig, 1835 & \\
\hline Lasiurus & xanthinus & Thomas, 1897 & \\
\hline Myotis & aelleni & Baud, 1979 & \\
\hline Myotis & albescens & (É. Geoffroy St.-Hilaire, 1806) & \\
\hline Myotis & atacamensis & (Lataste, 1892) & \\
\hline Myotis & auriculus & Baker \& Stains, 1955 & \\
\hline Myotis & californicus & (Audubon \& Bachman, 1842) & \\
\hline Myotis & carteri & LaVal, 1973 & 12 \\
\hline Myotis & caucensis & J. A. Allen, 1914 & 71 \\
\hline Myotis & chiloensis & (Waterhouse, 1840) & \\
\hline Myotis & cobanensis & Goodwin, 1955 & \\
\hline Myotis & diminutus & Moratelli \& Wilson, 2011 & 69 \\
\hline Myotis & dinellii & Thomas, 1902 & 68 \\
\hline Myotis & dominicensis & Miller, 1902 & \\
\hline Myotis & elegans & Hall, 1962 & \\
\hline Myotis & fortidens & Miller \& Allen, 1928 & \\
\hline Myotis & handleyi & Moratelli et al., 2013 & 71 \\
\hline Myotis & izecksohni & Moratelli et al., 2011 & 70 \\
\hline Myotis & keaysi & J. A. Allen, 1914 & \\
\hline Myotis & lavali & Moratelli et al., 2011 & 70 \\
\hline Myotis & levis & (I. Geoffroy St.-Hilaire, 1824) & 68 \\
\hline Myotis & martiniquensis & LaVal, 1973 & 57 \\
\hline Myotis & nesopolus & Miller, 1900 & 57 \\
\hline Myotis & nigricans & (Schinz, 1821) & $12,70,71$ \\
\hline Myotis & nyctor & LaVal \& Schwartz, 1974 & 57 \\
\hline Myotis & oxyotus & (W. Peters, 1866) & \\
\hline Myotis & riparius & Handley 1960 & \\
\hline Myotis & ruber & (É. Geoffroy St.-Hilaire, 1806) & \\
\hline
\end{tabular}

continúa.. 
(A) Los nombres de subfamilia "Glyphonycterinae" y "Rhinophyllinae" (sensu Baker et al. 2003) no están disponibles por no cumplir los requisitos del Código de Nomenclatura Zoológica (ICZN 1999).

\begin{tabular}{llll}
\hline Género & Especie & Autor y año & Referencias \\
\hline Myotis & simus & Thomas, 1901 & \\
Myotis & velifer & (J. A. Allen, 1890) & \\
Nycticeius & cubanus & Gundlach, 1861 & \\
Nycticeius & humeralis & (Rafinesque, 1818) & 45 \\
Perimyotis & subflavus & (F. Cuvier, 1832) & \\
Rhogeessa & aeneus & Goodwin, 1958 & 5 \\
Rhogeessa & bickhami & Baird et al., 2012 & \\
Rhogeessa & genowaysi & Baker, 1984 & \\
Rhogeessa & hussoni & Genoways \& Baker, 1996 & 4 \\
Rhogeessa & io & Thomas, 1903 & 5 \\
Rhogeessa & menchuae & Baird et al., 2012 & \\
Rhogeessa & minutilla & Miller, 1897 & \\
Rhogeessa & mira & LaVal, 1973 & \\
Rhogeessa & parvula & H. Allen, 1866 & 4 \\
Rhogeessa & tumida & H. Allen, 1866 & 4 \\
Rhogeessa & velilla & Thomas, 1903 & \\
\hline
\end{tabular}

La amplitud metodológica de los estudios muestra la divergencia de enfoques, y permite discutir los criterios empleados para alcanzar objetivos específicos o generales en cuanto a revisiones sistemáticas, pero también en la actualización de la taxonomía del orden Chiroptera (ver Vences et al. 2013). Cuando se aplican consistentemente, estos criterios pueden ayudar a producir estudios sistemáticos y taxonómicos robustos, como aquellos que se vienen realizando en roedores Sigmodontinos (Weksler et al. 2006; D'Elía y Pardiñas 2007). Otro resultado evidente de estas prácticas es la constante actualización de las listas de especies, a nivel local y regional (e. g. Brasil: Paglia et al. 2012, Colombia: Solari et al. 2013), y el uso cada vez más frecuente de caracteres morfológicos para identificar especies (e. g. Velazco y Gardner 2009).

El incremento en la aplicación de métodos filogenéticos usando datos moleculares ha mejorado la comprensión de la variación taxonómica para géneros en los cuales la morfología de ejemplares de museo era la única herramienta (tales como Artibeus, Larsen et al. 2007, 2010). La integración de información genética y cariotípica permite establecer qué variación morfológica tiene significado taxonómico (Baker y Bradley 2006; Baker et al. 2009), apoyando el punto de vista que sostiene que las prácticas taxonómicas sólidas requieren integrar múltiples datos al inferir límites específicos (de Queiroz 2007). En perspectiva, una filogenia bien soportada permite avanzar hipótesis sobre la diversificación y distribución de las especies en un género. Sin embargo, limitantes tales como identificaciones erróneas, o escaso muestreo geográfico o taxonómico, pueden afectar seriamente la interpretación de los resultados (ver Van Den Bussche y Weyandt 2003). Cuando los resultados incompletos se acumulan y comunican a mayor velocidad (por medios digitales en internet), el efecto puede ser confusión antes que resolución para los usuarios finales de esta información (e. g. museos, bases de datos, agencias de conservación, literatura general). 
Esta discusión (taxonomía contra nomenclatura) no es trivial. El hecho de reconocer a una entidad taxonómica como un sinónimo o una subespecie tiene consecuencias en áreas tan disimiles como la sistemática y la conservación, pues no puede aplicarse un mismo estado de amenaza a una subpoblación distinguible en términos genéticos (reproductivos) que a una población insuficientemente identificada. Por ejemplo, el primate Lagothrix lagothricha es una especie en situación de Amenaza según la UICN.

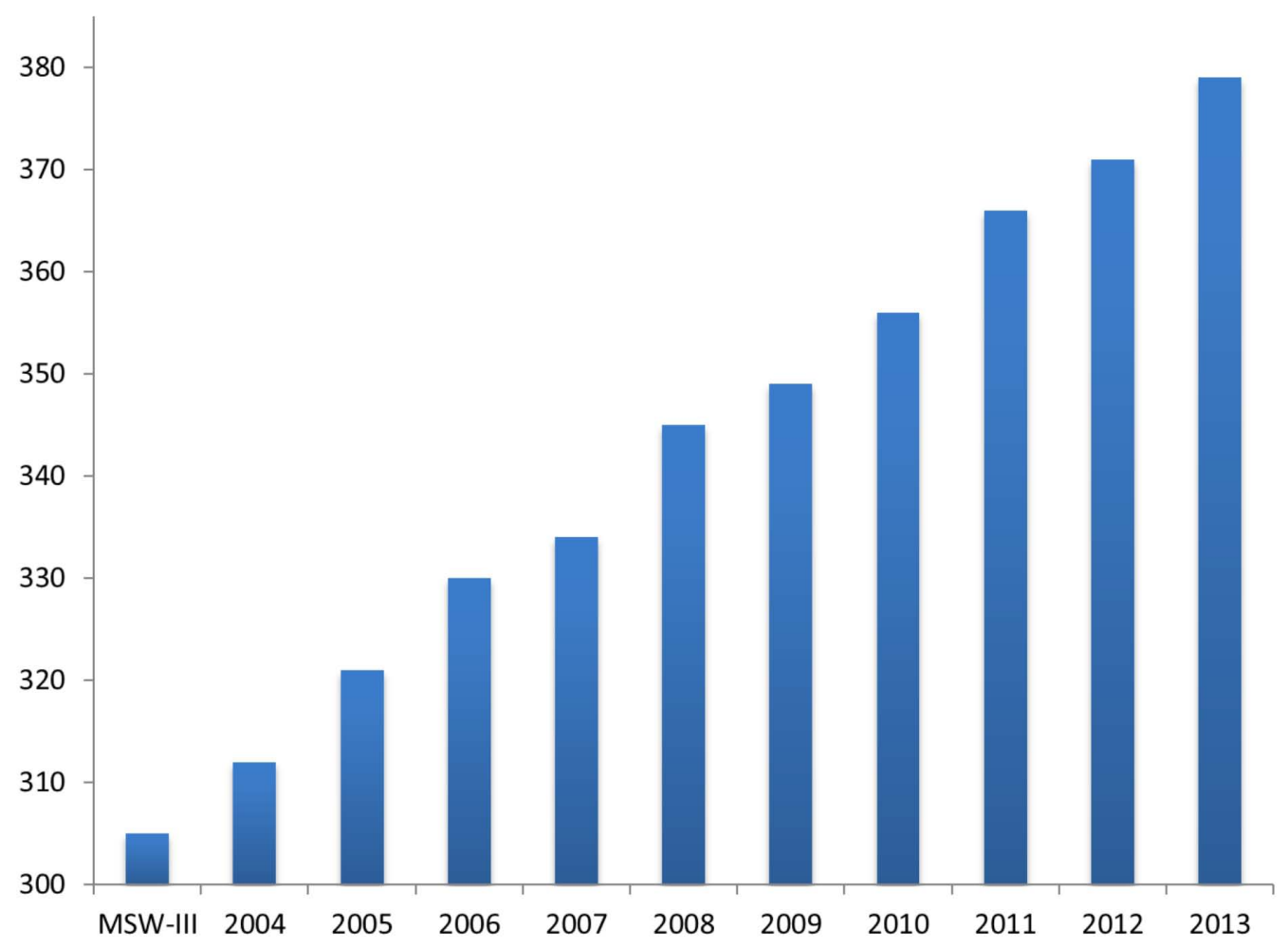

Figura 3. Incremento en el número total de especies válidas (al momento de publicación) de murciélagos Neotropicales a través del tiempo (en años). El punto de partida es el número de especies en Simmons (2005).

Sin embargo, debido a su compleja taxonomía, las poblaciones en Colombia pueden tomarse como Vulnerables (VU) en el caso de la subespecie L. lagothricha lagothricha, o Críticamente Amenazadas (CR) para L. lagothricha lugens (Defler 2010). Sin embargo, la reciente propuesta de distinguir subespecies de L. lugens endémicas de Colombia (Mantilla-Meluk 2013) puede crear un problema debido a la inestabilidad taxonómica asociada, aunque la propuesta refleje una aproximación integral para reconocer la diversidad real del grupo. Otros problemas pueden darse al cambiar drásticamente la nomenclatura, transfiriendo especies entre géneros, como en el caso de Artibeus incomitatus que se convierte en Dermanura watsoni (Solari et al., 2009), o sinonimizando nombres listados como especies amenazadas bajo nombres que no se han empleado por casi 50 años, como sucede al reemplazar Sturnira thomasi por S. angeli (Velazco y Patterson 2013), perdiendo de vista los motivos que estuvieron detrás de su inclusión en listas de amenaza o de su sinonimia.

Es importante notar que, para muchos de los usuarios finales de esta información, la falta Conclusiones de un consenso entre sistemáticos y la consecuente inestabilidad taxonómica debilita su confianza en los métodos y herramientas de estas disciplinas. Sin embargo, debe enfatizarse que el objetivo final de este tipo de estudios es facilitar la integración de 
datos genéticos, morfológicos, y biogeográficos para grupos de organismos (tales como especies o familias). Siendo la sistemática la disciplina que provee el método y el contexto para identificar relaciones entre organismos, es necesario expresar estas relaciones como una clasificación congruente con los nombres usados para los organismos, y que con ello se facilite la comunicación entre los investigadores y las demás partes interesadas. Igualmente, a largo plazo, este conocimiento permite identificar y describir taxa no reconocidos, mejorando nuestro entendimiento de la biodiversidad del planeta.

La diversidad de murciélagos Neotropicales se actualiza a 380 especies en 98 géneros, lo que representa un incremento neto de 75 especies respecto a lo presentado por Simmons (2005), a una tasa aproximada de 8 especies/año. El uso extensivo de métodos y criterios filogenéticos para identificar géneros o especies monofiléticos ha permitido un mejor entendimiento de estas relaciones, de sus distribuciones y finalmente de su diversificación (e. g. Baker et al. 2003). Estos cambios se han dado en al menos 34 géneros, ligeramente mayor a un tercio del total de géneros reconocidos para la región.

Durante el proceso de revisión del manuscrito se describió una nueva especie en el género Thyroptera (Velazco et al. 2014), y un nuevo género y tribu en Lonchophyllinae (Hsunycteris, Parlos et al. 2014). Si a este total agregamos el número de especies reconocidas pero no descritas formalmente, al menos cinco más, y las reconocidas como extintas (1) o fósiles (3), el total se acerca a 390 especies. En general, estos cambios muestran que los avances continuarán a medida que se incrementa la exploración e investigación, así como la integración de múltiples fuentes de información, asociando estudios morfológicos, datos moleculares, cariotipos, e historia natural, brindando una mayor certeza y soporte en los resultados, y (esperamos) mayor aceptación de los mismos entre los investigadores del Neotrópico.

\section{Agradecimientos}

El primer autor (SS) agradece a la Universidad de Antioquia por su apoyo para desarrollar esta y otras investigaciones en el área de mamíferos. La mayoría de la información presentada en este artículo proviene de intensas discusiones con colegas que comparten sus intereses o dudas en temas de sistemática y taxonomía, y en Chiroptera en particular. Otros han compartido sus experiencias y han permitido un mejor conocimiento de muchas familias y géneros. Por ello, agradecemos especialmente a R. J. Baker (Texas Tech University), A. L. Gardner (National Museum of Natural History), B. D. Patterson (Field Museum of Natural History), P. M. Velazco (American Museum of Natural History), y D. Zurc (Universidad de Antioquia). Finalmente, agradecemos a R. Owen (editor especial de este volumen) y a tres evaluadores anónimos que ayudaron a mejorar significativamente este manuscrito.

\section{Literatura citada}

NOTA: la numeración en las referencias está en función de la Tabla 2.

[1] Agapow, P. M., O. R. P. Bininda-Emonds, K. A. Crandall, J. L. Gittleman, G. M. Mace, J. C. Marshall, y A. Purvis. 2004. The impact of species concepts on biodiversity studies. The Quarterly Review of Biology 79:161-179. 
[2] Albuja, V. L., y A. L. Gardner. 2005. A new species of Lonchophylla Thomas (Chiroptera: Phyllostomidae) from Ecuador. Proceedings of the Biological Society of Washington 118:442-449.

[3] Ammerman, L. K., D. N. Lee, y M. Tipps. 2012. First molecular phylogenetic insights into the evolution of free-tailed bats in the subfamily Molossinae (Molossidae, Chiroptera). Journal of Mammalogy 93:12-28.

[4] Baird, A. B., D. M. Hilus, J. C. Patton, y J. W. Bickham. 2008. Evolutionary history of the genus Rhogeessa (Chiroptera: Vespertilionidae) as revealed by mitochondrial DNA sequences. Journal of Mammalogy 89:744-754.

[5] Baird, A. B., M. R. Marchán-Rivadeneira, S. G. Perez, y R. J. Baker. 2012. Morphological analysis and description of two new species of Rhogeessa (Chiroptera: Vespertilionidae) from the Neotropics. Occasional Papers, Museum of Texas Tech University 307:1-25.

[6] Baker, R. J., y R. D. Bradley. 2006. Speciation in mammals and the genetic species concept. Journal of Mammalogy 87:643-662.

[7] Baker, R. J., C. A. Porter, J. C. Patton, y R. A. Van Den Bussche. 2000. Systematics of bats of the family Phyllostomidae based on RAG2 DNA sequences. Occasional Papers, Museum of Texas Tech University 202:1-16.

[8] Baker, R. J., S. R. Hoofer, C. A. Porter, y R. A. Van Den Bussche. 2003. Diversification among New World Leaf-Nosed Bats: an evolutionary hypothesis and classification inferred from digenomic congruence of DNA sequence. Occasional Papers, Museum of Texas Tech University 230:1-32.

[9] Baker, R. J., R. M. Fonseca, D. A. Parish, C. J. Phillips, y F. G. Hoffmann. 2004. New bat of the genus Lophostoma (Phyllostomidae: Phyllostominae) from northwestern Ecuador. Occasional Papers, Museum of Texas Tech University 232:1-16.

[10] Baker, R. J., M. M. McDonough, V. J. Swier, P. A. Larsen, J. P. Carrera, Y L. K. Ammerman. 2009. New species of bonneted bat, genus Eumops (Chiroptera: Molossidae) from the lowlands of western Ecuador and Peru. Acta Chiropterologica 11:1-13.

[11] Barraclough, T. G., y S. Nee. 2001. Phylogenetics and speciation. Trends in Ecology and Evolution 26:391-399.

[12] Ceballos, G., y J. Arroyo-Cabrales. 2012. Lista actualizada de los mamíferos de México 2012. Revista Mexicana de Mastozoología, nueva época 2: 27-80.

[13] Ceballos, G., y P. R. Ehrlich. 2009. Discoveries for new mammal species and their implications for conservation and ecosystem services. Proceedings of the National Academy of Sciences, USA 106:3841-3846.

[14] Clare, E. L., A. M. Adams, A. Z. Maya-Simoes, J. L. Eger, P. D. Hebert, y M. B. Fenton. 2013. Diversification and reproductive isolation: cryptic species in the only New World high-duty cycle bat, Pteronotus parnellii. BMC Evolutionary Biology 13:26.

[15] Cracraft, J. 1983. Species concepts and speciation analysis. Current Ornithology 1:159-187.

[16] Cuartas-Calle, C. A., J. Muñoz, y M. González. 2001. Una nueva especie de Carollia Gray, 1838 (Chiroptera: Phyllostomidae) de Colombia. Actualidades Biológicas 23:63-73. 
[17] D’Elía, G., y U. F. J. Pardiñas. 2007. Putting names to the phylogenetic diversity of Neotropical sigmodontine rodents: new genera for known species. Mammalia 71:143-145.

[18] Dávalos, L. M. 2004. A new Chocoan species of Lonchophylla (Chiroptera: Phyllostomidae). American Museum Novitates 3426:1-16.

[19] Dávalos, L. M. 2005. Molecular phylogeny of Funnel-eared bats (Chiroptera: Natalidae), with notes on biogeography and conservation. Molecular Phylogenetics and Evolution 37:91-103.

[20] Dávalos, L. M. 2006. The geography of diversification in the mormoopids (Chiroptera: Mormoopidae). Biological Journal of the Linnean Society 88:101-118.

[21] Dávalos, L. M., y A. Corthals. 2008. A new species of Lonchophylla (Chiroptera: Phyllostomidae) from the eastern Andes of northwestern South America. American Museum Novitates 3635:1-16.

[22] Dayrat, B. 2005. Towards integrative taxonomy. Biological Journal of the Linnean Society 85:407-415.

[23] De Queiroz, K. 1998. The general lineage concept of species, species criteria, and the process of speciation: A conceptual unification and terminological recommendations. Pp. 57-75 en Endless forms: Species and speciation (Howard, D. J., y S. H. Berlocher, eds.). Oxford University Press. New York, EE.UU.

[24] De Quelroz, K. 2007. Species concepts and species delimitation. Systematic Biology 56:879-886.

[25] Defler, T. R. 2010. Historia natural de los Primates Colombianos. Universidad Nacional de Colombia. Bogotá, Colombia.

[26] Dias, D., C. D. L. Esberard, y R. Moratelli. 2013. A new species of Lonchophylla (Chiroptera, Phyllostomidae) from the Atlantic Forest of southeastern Brazil, with comments on L. bokermanni. Zootaxa 3722:347-360.

[27] Drexler, J. F., V. M. Corman, M. A. Müller, G. D. Maganga, P. Vallo, T. Binger, F. Gloza-Rausch, A. Rasche, S. Yordanov, A. Seebens, S. Oppong, Y. A. Sarkodie, C. Pongombo, A. N. Lukashev, J. Schmidt-Chanasit, A. Stöcker, A. J. Borges Carneiro, S. Erbar, A. Maisner, F. Fronhoffs, R. Buettiner, E. K. V. Kalko, T. Kruppa, C. R. Franke, R. Kallies, E. R. N. Yandoko, G. Herrler, C. Reusken, A. Hassanin, D. H. Krüger, S. Matthee, R. G. Ulrich, E. M. Leroy, y C. Drosten. 2012. Bats hosts major mammalian Paramyxoviruses. Nature Communications 3:796.

[28] Eger, J. L. 2008. Family Molossidae. Pp. 399-439 en Mammals of South America. Volume 1. Marsupials, Xenarthrans, Shrews, and Bats (Gardner, A. L., ed.). The University of Chicago Press. Chicago, EE.UU.

[29] FonseCA, R. M., y C. M. Pinto. 2004. A new Lophostoma (Chiroptera: Phyllostomidae: Phyllostominae) from the Amazonia of Ecuador. Occasional Papers, Museum of Texas Tech University 242:1-9.

[30] Fonseca, R. M., S. R. Hoofer, C. A. Porter, C. A. Cline, D. A. Parish, F. G. Hoffmann, Y R. J. BAKER. 2007. Morphological and molecular variation within little bigeared bats of the genus Micronycteris (Phyllostomidae: Micronycterinae) from San Lorenzo, Ecuador. Pp. 721-746 en The Quintessential Naturalist: Honoring the Life and Legacy of Oliver P. Pearson (Kelt, D. A., E. P. Lessa, J. Salazar-Bravo, y J. L. 
Patton, eds.). University of California, Publications in Zoology 134. University of California Press. Berkeley, EE.UU.

[31] Garbino, G. S. y T. A. Tejedor. 2013. Natalus macrourus (Gervais, 1856) (Chiroptera: Natalidae) is a senior synonym of Natalus espiritosantensis (Ruschi, 1951). Mammalia 77:237-240.

[32] Gardner, A. L. 2008. Tribe Sturnirini. Pp. 363-376 en Mammals of South America. Volume 1. Marsupials, Xenarthrans, Shrews, and Bats (Gardner, A. L., ed.). The University of Chicago Press. Chicago, EE.UU.

[33] González-Ruiz, N., J. Ramírez-Pulido, y J. Arroyo-Cabrales. 2011. A new species of mastiff bat (Chiroptera: Molossidae: Molossus) from Mexico. Mammalian Biology 76:461-469.

[34] Gregorin, R., y A. D. Ditchfield. 2005. New genus and species of nectar-feeding bat in the tribe Lonchophyllini (Phyllostomidae: Glossophaginae) from northeastern Brazil. Journal of Mammalogy 86:403-14.

[35] Gregorin, R., y E. A. Chiquito. 2010. Revalidation of Promops davisoni Thomas (Molossidae). Chiroptera Neotropical 16:648-660.

[36] Gregorin, R., E. Goncalyes, B. K. Lim, y M. D. Engstrom. 2006. New species of diskwinged bat Thyroptera and range extension for T. discifera. Journal of Mammalogy 87:238-246.

[37] Griffiths, T. A., y A. L. Smith. 1991. Systematics of emballonuroid bats (Chiroptera: Emballonuridae and Rhinopomatidae) based on hyoid morphology. Bulletin of the American Museum of Natural History 206:62-83.

[38] Griffiths, T. A., y A. L. Gardner. 2008. Subfamily Glossophaginae Bonaparte, 1845. Pp. 224-244 en Mammals of South America. Volume 1. Marsupials, Xenarthrans, Shrews, and Bats (Gardner, A. L., ed.). The University of Chicago Press. Chicago, EE.UU.

[39] Groves, C. P. 2001. Primate Taxonomy. Smithsonian Institution Press. Washington, EE.UU.

[40] Groves, C. P., y P. Grubb. 2011. Ungulate Taxonomy. The Johns Hopkins University Press. Baltimore, EE.UU.

[41] Gutierrez, E. E., y J. Molinari. 2008. Morphometrics and taxonomy of bats of the genus Pteronotus (subgenus Phyllodia) in Venezuela. Journal of Mammalogy 89:292-305.

[42] Handley, C. O., Jr., y A. L. Gardner. 2008. Genus Histiotus P. Gervais, 1856. Pp. 450-457 in Mammals of South America. Volume 1. Marsupials, Xenarthrans, Shrews, and Bats (Gardner, A. L., ed.). The University of Chicago Press. Chicago, EE.UU.

[43] Hoofer, S. R., y R. A. Van Den Bussche. 2003. Molecular phylogenetics of the chiropteran family Vespertilionidae. Acta Chiropterologica 5 (suppl.):1-63.

[44] Hoofer, S. R., y R. J. BaKer. 2006. Molecular systematic of Vampyressine bats (Phyllostomidae: Stenodermatinae) with comparison of direct and indirect surveys of mitochondrial DNA variation. Molecular Phylogenetics and Evolution 39:424438. 
[45] Hoofer, S. R., R. A. Van Den Bussche, y I. Horacek. 2006. Generic status of the American pipistrelles (Vespertilionidae) with description of a new genus. Journal of Mammalogy 87: 981-992.

[46] Hoofer, S. R., S. Solari, P. A. Larsen, R. D. Bradley, y R. J. Baker. 2008. Phylogenetics of the fruit-eating bats (Phyllostomidae: Artibeina) inferred from mitochondrial DNA sequences. Occasional Papers, Museum of Texas Tech University 277:1-15.

[47] International Commission of Zoological Nomenclature (ICZN). 1999. The International Code of Zoological Nomenclature, cuarta edición. International Trust for Zoological Nomenclature. Londres, Inglaterra.

[48] Jarrin-V., P, YT. H. Kunz. 2011. A new species of Sturnira (Chiroptera: Phyllostomidae) from the Chocó forest of Ecuador. Zootaxa 2755:1-35.

[49] Jones, J. K., JR., Y C. S. Hood. 1993. Synopsis of South American bats of the family Emballonuridae. Occasional Papers of the Museum of Texas Tech University 155:1-32.

[50] Jones, G., D. S. Jacobs, T. H. Kunz, M. R. Willig, y P. A. Racey. 2009. Carpe noctem: the importance of bats as bioindicators. Endangered Species Research 8:93-115.

[51] Koopman, K. F. 1984. A synopsis of the families of bats, Part VII. Bat Research News 25:25-27.

[52] Koopman, K. F. 1994. Chiroptera: Systematics. Handbook of Zoology: Mammalia, vol. 8. W. de Gruyter \& Co. Berlín, Alemania.

[53] Kunz, T. H., E. Braun de Torrez, D. Bauer, T. Lobova, y T. H. Fleming. 2011. Ecosystem services provided by bats. Annals of the New York Academy of Sciences 1223:138.

[54] Larsen, P. A., S. R. Hoofer, M. C. Bozeman, S. C. Pedersen, H. H. Genoways, C. J. Phillips, D. E. Pumo, y R. J. Baker. 2007. Phylogenetics and phylogeography of the Artibeus jamaicensis complex based on cytochrome- $b$ DNA sequences. Journal of Mammalogy 88:712-727.

[55] Larsen, P. A., M. R. Marchán-Rivadeneyra, y R. J. Baker. 2010. Taxonomic status of Andersen's fruit-eating bat (Artibeus jamaicensis aequatorialis) and revised classification of Artibeus (Chiroptera: Phyllostomidae). Zootaxa 2648:45-60.

[56] Larsen, P. A., L. Siles, S. C. Pedersen, y G. G. Kwiecinski. 2011. A new species of Micronycteris (Chiroptera: Phyllostomidae) from Saint Vincent, Lesser Antilles. Mammalian Biology 76:687-700.

[57] Larsen, R. J., P. A. Larsen, H. H. Genoways, F. M. Catzeflis, K. Geluso, G. G. Kwiecinski, S. C. Pedersen, F. Simal, y R. J. Baker. 2012. Evolutionary history of Caribbean species of Myotis, with evidence of a third Lesser Antillean endemic. Mammalian Biology 77:124-134.

[58] Lim, B. K., M. D. Engstrom, J. C. Patton, y J. W. Bickham. 2008a. Systematic review of small fruit-eating bats (Artibeus) from the Guianas, and a re-evaluation of $A$. glaucus bogotensis. Acta Chiropterologica 10:243-256.

[59] Lim, B. K., M. D. Engstrom, J. W. Bickham, y J. C. Patton. 2008b. Molecular Phylogeny of New World Sheath-tailed Bats (Emballonuridae: Diclidurini) based on loci from the four genetic transmission systems in mammals. Biological Journal of the Linnean Society 93:189-209. 
[60] Lim, B. K., M. D. Engstrom, F. A. Reid, N. B. Simmons, R. S. Voss, y D. W. Fleck. 2010. A new species of Peropteryx (Chiroptera: Emballonuridae) from western Amazonia with comments on phylogenetic relationships within the genus. American Museum Novitates 3686:1-20.

[61] López-González, C., y S. J. Presley. 2001. Taxonomic status of Molossus bondae J.A. Allen, 1904. Journal of Mammalogy 82:760-774.

[62] López-Wilchis, R., L. M. Guevara-Chumacero, N. A. Pérez, J. Juste, C. Ibáñez, y D. L. A. Barriga Sosa. 2012. Taxonomic status assessment of the Mexican populations of funnel-eared bats, genus Natalus (Chiroptera: Natalidae). Acta Chiropterologica 14:305-316.

[63] Mantilla-Meluk, H. 2013. Subspecific variation: an alternative biogeographic hypothesis explaining variation in coat color and cranial morphology in Lagothrix lugens (Primates: Atelidae) from Colombia. Primate Conservation 26:33-48.

[64] Mantilla-Meluk, H., y R. J. Baker. 2006. Systematics of small Anoura (Chiroptera: Phyllostomidae) from Colombia, with description of a new species. Ocassional Papers, Museum of Texas Tech University 261:1-18.

[65] Mantilla-Meluk, H., y R. J. Baker. 2010. New species of Anoura (Chiroptera: Phyllostomidae) from Colombia, with systematic remarks and notes on the distribution of the A. geoffroyi complex. Occasional Papers, Museum of Texas Tech University 292:1-19.

[66] McCarthy, T. J., L. Albuja, y M. Alberico. 2006. A new species of chocoan Sturnira (Chiroptera: Phyllostomidae: Stenodermatinae) from western Ecuador and Colombia. Annals of Carnegie Museum 75:97-110.

[67] Miranda, J. M. D., I. P. Bernardi, y F. C. Passos. 2006. A new species of Eptesicus (Mammalia: Chiroptera: Vespertilionidae) from the Atlantic Forest, Brazil. Zootaxa 1383:57-68.

[68] Miranda, J. M. D., I. P. Bernardi, J. Sponchiado, y F. C. Passos. 2013. The taxonomic status of Myotis levis levis and Myotis levis dinelli (Mammalia: Chiroptera: Vespertilionidae). Zoologia 30: 513-518.

[69] Moratelli, R., y D. E. Wilson. 2011. A new species of Myotis, Kaup 1829 (Chiroptera: Vespertilionidae) from Ecuador. Mammalian Biology 76:608-614.

[70] Moratelli, R., A. L. Peracchi, D. Dias, y J. A. De Oliveira. 2011. Geographic variation in South American populations of Myotis nigricans (Schinz, 1821) (Chiroptera, Vespertilionidae), with description of two new species. Mammalian Biology 76:592-607.

[71] Moratelli, R., A. L. Gardner, J. A. de Oliveira, D. E. Wilson. 2013. Review of Myotis (Chiroptera, Vespertilionidae) from northern South America, including description of a new species. American Museum Novitates 3780:1-36.

[72] Muchinala N., P. Mena V., y L. Albuja V. 2005. A new species of Anoura (Chiroptera: Phyllostomidae) from the Ecuadorian Andes. Journal of Mammalogy 86:457-461.

[73] Muñoz, J., C. A. Cuartas-Calle, y M. González. 2004. Se describe una nueva especie de murciélago del género Carollia Gray, 1838 (Chiroptera: Phyllostomidae) de Colombia. Actualidades Biológicas 26:80-90. 
[74] Nogueira, M. R., I. P. Lima, A. L. Peracchi, y N. B. Simmons. 2012. New genus and species of nectar-feeding bat from the Atlantic forest of southeastern Brazil (Chiroptera: Phyllostomidae: Glossophaginae). American Museum Novitates 3747:1-30.

[75] Ochoa, J. G., y J. Sánchez H. 2005. Taxonomic status of Micronycteris homezi (Chiroptera, Phyllostomidae). Mammalia 69:323-336.

[76] Ortega, J., y H. T. Arita. 1998. Neotropical-Neartic limits in Middle America as determined by distribution of bats. Journal of Mammalogy 79:772-783.

[77] Pacheco, V., S. Solari, y P. M. Velazco. 2004. A new species of Carollia (Chiroptera: Phyllostomidae) from the Andes of Peru and Bolivia. Occasional Papers, Museum of Texas Tech University 236:1-15.

[78] Padial, J. M., A. Miralles, I. De la Riva, y M. Vences. 2010. The integrative future of taxonomy. Frontiers in Zoology 7:16.

[79] Paglia, A. P., G. A. B. da Fonseca, A. B. Rylands, G. Herrmann, L. M. S. Aguiar, A. G. Chiarello, Y. L. R. Leite, L. P. Costa, S. Siciliano, M. C. M. Kierulff, S. L. Mendes, V. da C. Tavares, R. A. Mittermeier, y J. L. Patton. 2012. Lista Anotada dos Mamíferos do Brasil / Annotated Checklist of Brazilian Mammals. Occasional Papers in Conservation Biology 6:1-76.

[80] Parlos, J. A., R. M. Timm, V. J. Swier, H. Zeballos, y R. J. Baker. 2014. Evaluation of paraphyletic assemblages within Lonchophyllinae, with description of a new tribe and genus. Occasional Papers, Museum of Texas Tech University 320:1-23.

[81] Patterson, B. D. 1996. The 'species alias' problem. Nature 380:589.

[82] Patterson, B. D. 2000. Patterns and trends in the discovery of new Neotropical mammals. Diversity \& Distributions 6:145-151.

[83] Porter, C. A., S. R. Hoofer, C. A. Cuine, F. G. Hoffmann, y R. J. Baker. 2007. Molecular phylogenetics of the phyllostomid bat genus Micronycteris with description of two new subgenera. Journal of Mammalogy 88:1205-1215.

[84] Reeder, D. M., K. M. Helgen, y D. E. Wilson. 2007. Global trend and biases in new mammal species discoveries. Occasional Papers, Museum of Texas Tech University 269:1-35.

[85] Sánchez-Hernández, C., M. L. Romero-Almaraz, y G. D. Schnell. 2005. New species of Sturnira (Chiroptera: Phyllostomidae) from northern South America. Journal of Mammalogy 86:866-872.

[86] Siles, L., D. M. Brooks, H. Aranibar, T. Tarifa, R. J. Vargas M., J. M. Rojas, y R. J. Baker. 2013. A new species of Micronycteris (Chiroptera: Phyllostomidae) from Bolivia. Journal of Mammalogy 94:881-896.

[87] Simmons, N. B. 2005. Order Chiroptera. Pp: 312-529 en Mammal Species of the World: a taxonomic and geographic reference, tercera edición (Wilson, D. E., y D. M. Reeder, eds.). The Johns Hopkins University Press. Baltimore, EE.UU.

[88] Soları, S., y R. J. Baker. 2006. Mitochondrial DNA sequence, karyotypic, and morphological variation in the Carollia castanea species complex (Chiroptera: Phyllostomidae) with description of a new species. Occasional Papers, Museum of Texas Tech University 254:1-16. 
[89] Solari, S., y R. J. Baker. 2007. Book Review: "Wilson, D. E., and D. M. Reeder (eds.). 2005. Mammal species of the World: a taxonomic and geographic reference". Journal of Mammalogy 88:824-830.

[90] Solari, S., S. R. Hoofer, P. A. Larsen, A. D. Brown, R. J. Bull, J. A. Guerrero, J. Ortega, J. P. Carrera, R. D. Bradley, y R. J. Baker. 2009. Operational criteria for genetically defined species: analysis of the diversification of the small fruit-eating bats, Dermanura (Phyllostomidae: Stenodermatinae). Acta Chiropterologica 11:279-288.

[91] Solari, S., P. M. Velazco, y B. D. Patterson. 2012. Hierarchical organization of Neotropical mammal diversity and its historical basis. Pp. 145-156 en Bones, clones, and biomes: the history and geography of recent Neotropical mammals (Patterson, B. D., y L.P. Costa, eds.). The University of Chicago Press. Chicago, EE.UU.

[92] Solari, S., Y. Muñoz-Saba, J. V. Rodríguez-Mahecha, T. Defler, H. Ramírez-Chaves, y F. Trujillo. 2013. Diversidad, Endemismo y Conservación de los Mamíferos de Colombia. Mastozoología Neotropical 20:301-365.

[93] Springer, M. S., E. C. Teeling, O. Madsen, M. J. Stanhope, y W. W. de Jong. 2001. Integrated fossil and molecular data reconstruct bat echolocation. Proceedings of the National Academy of Sciences 98:6241-6246.

[94] Taddeı, V. A., Y B. K. Lım. 2010. A new species of Chiroderma (Chiroptera, Phyllostomidae) from northeastern Brazil. Brazilian Journal of Biology 70:381386.

[95] Teeling, E. C., O. Madsen, M. J. Stanhope, W. W. de Jong, R. A. Van Den Bussche, y M. S. Springer. 2002. Microbat paraphyly and the convergent evolution of a key innovation in Old World Rhinolophoid microbats. Proceedings of the National Academy of Sciences 99:1432-1436.

[96] Teeling, E. C., O. Madsen, W. J. Murphy, M. S. Springer, y J. O’'Brien. 2003. Nuclear gene sequences confirm an ancient link between New Zealand's short-tailed bat and South American Noctilionoid bats. Molecular Phylogenetics and Evolution 28:308-319.

[97] Teeling, E. C., M. S. Springer, O. Madsen, P. Bates, J. O'Brien, y W. J. Murphy. 2005. A molecular phylogeny for bats illuminates biogeography and the fossil record. Science 307:580-584.

[98] Tejedor, A. 2005. A new species of funnel-ear bat (Natalidae: Natalus) from Mexico. Journal of Mammalogy 86:1109-1120.

[99] Tejedor, A. 2006. The type locality of Natalus stramineus (Chiroptera: Natalidae): implications for the taxonomy and biogeography of the genus Natalus. Acta Chiropterologica 8:361-380.

[100] Tejedor, A. 2011. Systematics of funnel-eared bats (Chiroptera: Natalidae). Bulletin of the American Museum of Natural History 353:1-140.

[101] Tıмm, R. M., y H. H. Genoways. 2004. The Florida bonneted bat, Eumops floridanus (Chiroptera: Molossidae): distribution, morphometrics, systematics and ecology. Journal of Mammalogy 85:852-865. 
[102] Tong, S., X. Zhu, Y. Lı, M. Shi, J. Zhang, M. Bourgeois, H. Yang, X. Chen, S. Recuenco5, J. Gomez, L.-M. Chen, A. Johnson, Y. TaO, C. Dreyfus, W. Yu, R. McBride, P. J. Carney, A. T. Gilbert, J. Chang, Z. Guo, C. T. Davis, J. C. Paulson, J. Stevens, C. E. Rupprecht, E. C. Holmes, I. A. Wilson, y R. O. Donis. 2013. New World bats harbor diverse Influenza a viruses. PLOS Pathogens 9:e1003657.

[103] Van Den Bussche, R. A. y S. E. Weyandt. 2003. Mitochondrial and nuclear DNA sequence data provide resolution to sister-group relationship within Pteronotus (Chiroptera: Mormoopidae). Acta Chiropterologica 5:1-13.

[104] Van Den Bussche, R. A., y S. R. Hoofer. 2004. Phylogenetic relationships among recent Chiropteran families and the importance of choosing appropriate out-group taxa. Journal of Mammalogy 85:321-330.

[105] Velasco, J. D. 2009. When monophyly is not enough: exclusivity as the key to defining a phylogenetic species concept. Biology and Philosophy 24:473-486.

[106] Velazco, P. M. 2005. Morphological Phylogeny of the bat genus Platyrrhinus Saussure, 1860 (Chiroptera: Phyllostomidae) with the description of four new species. Fieldiana, Zoology, new series 105:1-54.

[107] Velazco, P. M., y R. Cadenillas. 2011. On the identity of Lophostoma silvicolum occidentalis (Davis \& Carter, 1978) (Chiroptera: Phyllostomidae). Zootaxa 2962:120.

[108] Velazco, P. M., y A. L. Gardner. 2009. A new species of Platyrrhinus (Chiroptera: Phyllostomidae) from western Colombia and Ecuador, with emended diagnoses of P. aquilus, $P$. dorsalis, and $P$. umbratus. Proceedings of the Biological Society of Washington 122:249-281.

[109] Velazco, P. M., y A. L. Gardner. 2012. A new species of Lophostoma d'Orbigny 1836 (Chiroptera: Phyllostomidae) from Panama. Journal of Mammalogy 93:605614.

[110] Velazco, P. M., y B. D. Patterson. 2008. Phylogenetics and biogeography of the broad-nosed bats, genus Platyrrhinus (Chiroptera: Phyllostomidae). Molecular Phylogenetics and Evolution 49:749-759.

[111] Velazco, P. M., y B. D. Patterson. 2013. Diversification of yellow-shouldered bats, genus Sturnira (Chiroptera: Phyllostomidae), in the New World tropics. Molecular Phylogenetics and Evolution 159:785-812.

[112] Velazco, P. M., y N. B. Simmons. 2011. Systematics and taxonomy of great stripedfaced bats of the genus Vampyrodes Thomas, 1900 (Chiroptera: Phyllostomidae). American Museum Novitates 3710:1-35.

[113] Velazco, P. M., y S. Soları. 2003. Taxonomía de Platyrrhinus dorsalis y Platyrrhinus lineatus (Chiroptera: Phyllostomidae) en Perú. Mastozoología Neotropical 10:303319.

[114] Velazco, P. M., A. L. Gardner, y B. D. Patterson. 2010. Systematics of the Platyrrhinus helleri species complex (Chiroptera: Phyllostomidae), with descriptions of two new species. Zoological Journal of the Linnean Society 159:785-812.

[115] Velazco, P. M., R. Gregorin, R. S. Voss, y N. B. Simmons. 2014. Extraordinary local diversity of disk-winged bats (Thyropteridae: Thyroptera) in northeastern Peru, with the description of a new species and comments on roosting behavior. American Museum Novitates 3795:1-28. 
[116] Vences, M., J. M. Guayasamin, A. Miralles, y I. De la Riva. 2013. To name or not to name: criteria to promote economy of change in Linnean classification schemes. Zootaxa 3636:201-244.

[117] Weksler, M., A. R. Percequillo, y R. S. Voss. 2006. Ten new genera of Oryzomyine rodents (Cricetidae: Sigmodontinae). American Museum Novitates 3537:1-29.

[118] Wetterer, A. L., M. V. Rockman, y N. B. Simmons. 2000. Phylogeny of phyllostomid bats (Mammalia: Chiroptera): data from diverse morphological systems, sex chromosomes, and restriction sites. Bulletin of the American Museum of Natural History 248:1-200.

[119] Wiley, E. O. Y B. S. Lieberman. 2011. Phylogenetics: Theory and practice of phylogenetic systematic. Wiley-Blackwell. New Jersey, EE.UU.

[120] Wilson, D. E. 2008. Genus Myotis Kaup, 1829. Pp. 468-481 en Mammals of South America. Volume 1. Marsupials, Xenarthrans, Shrews, and Bats (Gardner, A. L., ed.). The University of Chicago Press. Chicago, EE.UU.

[121] Wilson, D. E. y D. M. Reeder (Ed.). 2005. Mammal Species of the World. A Taxonomic and Geographic Reference, tercera edicion. The Johns Hopkins University Press. Baltimore, EE.UU.

[122] Woodman, N. 2007. New species of nectar-feeding bat, genus Lonchophylla, from Western Colombia and Western Ecuador (Mammalia: Chiroptera: Phyllostomidae). Proceedings of the Biological Society of Washington 120:340-358.

[123] Woodman, N., y R. M. Tıмm. 2006. Characters and phylogenetic relationships of nectar-feeding bats, with descriptions of new Lonchophylla from western South America (Mammalia: Chiroptera: Phyllostomidae: Lonchophyllini). Proceedings of the Biological Society of Washington 119:437-476.

[124] Zachos, F. E., M. Apollonio, E. V. Bärmann, M. Festa-Bianchet, U. Göhlich, J. C. Habel, E. Haring, L. Kruckenhauser, S. Lovari, A. D. McDevitt, C. Pertoldi, G. E. Rössner, M. R. Sánchez-Villagra, M. Scandura, y F. Suchentrunk. 2013. Species inflation and taxonomic artifacts - a critical comment on recent trends in mammalian classification. Mammalian Biology 78:1-6.

[125] Zurc, D., y P. M. Velazco. 2010. Análisis morfológico y morfométrico de Carollia colombiana Cuartas et al. 2001 y C. monohernandezi Muñoz et al. 2004 (Phyllostomidae: Carollinae) en Colombia. Chiroptera Neotropical 16:567-572.

Sometido: 16 de diciembre de 2013

Revisado: 2 de marzo de 2014

Aceptado: 1 de abril de 2014

Editor asociado: Robert Owen

Diseño gráfico editorial: Gerardo Hernández 